\title{
Grain interaction mechanisms leading to intragranular orientation spread in tensile deformed bulk grains of interstitial-free steel
}

Winther, Grethe; Wright, Jonathan P.; Schmidt, Søren; Oddershede, Jette

Published in:

International Journal of Plasticity

Link to article, DOI:

10.1016/j.ijplas.2016.10.004

Publication date:

2017

Document Version

Peer reviewed version

Link back to DTU Orbit

Citation (APA):

Winther, G., Wright, J. P., Schmidt, S., \& Oddershede, J. (2017). Grain interaction mechanisms leading to intragranular orientation spread in tensile deformed bulk grains of interstitial-free steel. International Journal of Plasticity, 88, 108-125. https://doi.org/10.1016/j.jplas.2016.10.004

\section{General rights}

Copyright and moral rights for the publications made accessible in the public portal are retained by the authors and/or other copyright owners and it is a condition of accessing publications that users recognise and abide by the legal requirements associated with these rights.

- Users may download and print one copy of any publication from the public portal for the purpose of private study or research.

- You may not further distribute the material or use it for any profit-making activity or commercial gain

- You may freely distribute the URL identifying the publication in the public portal 


\title{
Grain interaction mechanisms leading to intragranular orientation spread in tensile deformed bulk grains of interstitial-free steel
}

\author{
Grethe Winther ${ }^{\mathrm{a},}{ }^{,}$, Jonathan P. Wright ${ }^{\mathrm{b}}$, Søren Schmidtc ${ }^{\mathrm{c}}$ and Jette Oddershede \\ aDepartment of Mechanical Engineering, Technical University of Denmark, 2800 \\ Kongens Lyngby, Denmark \\ bEuropean Synchrotron Radiation Facility, 38043 Grenoble, France \\ cDepartment of Physics, Technical University of Denmark, 2800 Kongens Lyngby, \\ Denmark
}

\begin{abstract}
The spatially resolved intragranular orientation spread in two representative bulk grains of interstitial-free steel deformed to $9 \%$ tension has been investigated. A three-dimensional X-ray diffraction microscopy experiment revealed that the two similarly oriented grains are both embedded in local environments representing the bulk texture, yet their deformation-induced rotations are very different. The ALAMEL model is employed to analyse the grain interaction mechanisms. Predictions of this model qualitatively agree with the directionality and magnitude of the experimental orientation spread. However, quantitative agreement requires fine-tuning of the boundary conditions. The majority of the modelled slip is accounted for by four slip systems also predicted to be active by the classical Taylor model in uniaxial tension, and most of the orientation spread along the grain boundaries is caused by relative variations in the activities of these. Although limited to two grains, the findings prove that shear at the grain boundaries as accounted for by the ALAMEL model is a dominant grain interaction mechanism.
\end{abstract}

\section{Keywords}

Grain boundaries (A); polycrystalline material (B); crystal plasticity (B); nondestructive evaluation (C); ALAMEL model 


\section{Introduction}

The deformation-induced evolution of crystallographic texture in metals has been a subject of research for decades due to its consequences for the properties of the metal, in particular mechanical anisotropy. By comparison of deformation textures measured by X-rays or neutrons with predictions of the earliest models of crystal plasticity by Sachs (Sachs, 1928) and Taylor (Taylor, 1938)/Bishop-Hill (Bishop and Hill, 1951), it became clear that these models in general produce too sharp textures. This deficiency is attributed to their basic assumptions that ignore complex interactions between the grains, which will also lead to intragranular orientation spreads.

Experimental studies of the orientation spread within individual grains observed at a surface became possible with the emergence of the technique of electron back scatter diffraction (EBSD), including in-situ deformation studies of the lattice rotations of surface grains (Allain-Bonasso et al., 2012; Chen et al., 2013; Di Gioacchino and Quinta Da Fonseca, 2015; Guery et al., 2016). Three dimensional data may be obtained by serial sectioning (Afrin et al., 2013; Lin et al., 2010), which is, however, destructive, implying that the dynamics of the grains cannot be monitored. By pressing two metal surfaces closely together during deformation, a three-dimensional environment of neighbouring grains has been mimicked while still enabling dynamic studies by intermittent separation and EBSD investigation of the surfaces (Bhattachayya et al., 2001; Quey et al., 2015, 2010).

By contrast, the method of three-dimensional X-ray diffraction (3DXRD) is capable of monitoring the dynamics of individual grains in their natural environment of neighbouring grains (Margulies et al., 2001; Poulsen et al., 2003). The technique can produce large maps of grain structures (Pokharel et al., 2014; Poulsen et al., 2001; Rollett et al., 2015; Schuren et al., 2015; Sørensen et al., 2012b), including crystallographic orientations and neighbour relations (Hefferan et al., 2009; Li and Suter, 2013; Schmidt et al., 2008), and also allows measurement of the elastic strain in each individual grain during deformation (Bernier et al., 2011; Oddershede et al., 2010). This gives a unique possibility of investigating the behaviour of individual grains and how they interact with their environment. Particular emphasis has been on understanding the deformation behaviour of metals by mapping intra-granular orientation gradients as a function of plastic deformation (Li et al., 2013; Lind et al., 2014; Pokharel et al., 2015; Toda et al., 2016) and measuring grain-resolved stresses associated with deformation twinning in hexagonal close packed metals (Abdolvand et al., 2015a, 2015b; Aydiner et al., 2009; Bieler et al., 2014; L. Wang et al., 2014), but also processes such as grain nucleation (West et al., 2009), growth (Poulsen et al., 2011; Schmidt et al., 2004, 2008) and coarsening (Dake et al., 2016; Sharma et al., 2012), crack evolution (Cerrone et al., 2015; Chatterjee et al., 2015; Oddershede et al., 2012; Ozturk et al., 2016), stress relaxation (Tang et al., 2015), creep (Schuren et al., 2015) and phase transformations (Barton and Bernier, 2012; Hedström et al., 2010; Offerman et al., 2006) have been investigated using 3DXRD. For completeness applications of 3DXRD to minerals (Borthwick et al., 2012; Hall and Wright, 2015; Sørensen et al., 2012a), deep earth science (Nisr et 
al., 2014, 2012; Rosa et al., 2015), nuclear materials (Brown et al., 2014; X. Zhang et al., 2015), superalloys (Sedmák et al., 2016) and ferroelectrics (Daniels et al., 2016; Majkut et al., 2016; Oddershede et al., 2015; Varlioglu et al., 2010) should also be mentioned.

In parallel with advances in the experimental studies of plastically deformed metals, polycrystal plasticity models have been improved to take grain interactions into account. Self-consistent polycrystal plasticity models were developed (Lebensohn and Tomé, 1993; Molinari et al., 1987), which take into account the interaction between all grains of a specific orientation with a homogenous matrix representing all the other grains. Recently, microstructurally derived hardening laws have been incorporated in selfconsistent models to study the combined effects of slip and twinning on texture evolution (Brown et al., 2012; Knezevic et al., 2013). According to the selfconsistent scheme, all grains of similar orientation are assumed to behave in the same way, but they are influenced by the initial and evolving texture.

Randomised fluctuations in stress (Leffers, 1979) or strain (Ma et al., 2004) or stress fluctuations derived from the stress states of neighbouring grains (Robert et al., 2004) were introduced to create scatter in the behaviour of otherwise similar grains. In addition, relaxed constraint models were developed, in which certain strain components are enforced and others allowed to fluctuate, based on the geometry of the grains (Kocks and Chandra, 1982). Inspired by these concepts, the interaction between two grains has been modelled by the LAMEL model (Van Houtte et al., 1999) with focus on the interaction across the large faces of flat and elongated grains. This model has since been generalized to the ALAMEL model (Van Houtte et al., 2005), in which the grain boundary plane is also introduced as a variable. Whereas the LAMEL and ALAMEL models consider the interaction between two grains, the GIA model (Crumbach et al., 2001) takes into account interactions between a cluster of grains. Additional cluster-type models have also been derived (Xie et al., 2014). Common to this class of models is that grains are paired for interaction studies on a statistical basis considering the initial texture and possibly also the experimental misorientation distribution across grain boundaries (Zhang et al., 2015).

More recently, advanced finite element (Roters et al., 2010; Zhang et al., 2016) or Fourier transformation (Eisenlohr et al., 2013; Lebensohn et al., 2012) based polycrystal plasticity models, which consider more detailed interactions, have been employed to simulate intragranular orientation spreads (Lebensohn et al., 2016; Quey et al., 2015). The grain structures used as input to these models differ in geometrical complexity, and assignment of neighbouring grains may be based on statistics or experimentally determined grain structures, either in 2D by EBSD or in 3D by synchrotron methods.

The combination of experiment and modelling has led to significant advances in our understanding of grain-scale behaviour, including effects of grain orientation (Pokharel et al., 2015; Winther et al., 2004), grain size (Allain-Bonasso et al., 2012) and shape (Delannay and Barnett, 2012), formation of grain boundary regions (Pokharel et al., 2015; Vachhani et al., 2016), directionality of 
intragranular orientation spread (Krog-Pedersen et al., 2009; Lebensohn et al., 2016) and the interaction of plastically soft and hard grains (Raabe et al., 2001).

The present study is a follow-up on a previous study, which characterised the intragranular orientation spread within selected grains of similar orientation in a 9\% tensile deformed interstitial-free steel (Oddershede et al., 2015) by 3DXRD and analysed this by Taylor/Bishop-Hill modelling. It was concluded that the orientation spread of the grains had the same overall directionality, which was attributed to unbalanced activation of a few slip systems. The present study adds the experimentally determined spatial distribution of the orientation spread in the deformed state as well as information about the neighbouring grains. This extension allows analysis of the grain interaction mechanisms. In particular, the aim is to investigate if co-operative shear at the grain boundaries, as assumed by the ALAMEL model, is an important and effective mechanism of grain interaction.

The ALAMEL model was originally derived as a statistical model. Subsequent comparison of the predictions of the ALAMEL model with a finite element-based crystal plasticity model, using a model microstructure of columnar grains with hexagonal cross sections, however, showed good agreement (Kanjarla et al., 2010). To the authors' knowledge, the present study is the first evaluation of the ALAMEL model by direct comparison to experimentally observed grains.

The ALAMEL model is chosen among the other cluster-type models because of its relative simplicity and focus on interactions by co-operative shear at a single boundary between two neighbouring grains. While the ALAMEL model allows for both grain boundary shears and strain partitioning between the two grains, the boundary conditions in terms of the average strain of the two grains in the boundary region is required as input. In the present study this disadvantage is circumvented by a trial-and-error approach to determine boundary conditions that agree with the observed experimental data within reasonable limits. However, identification of the exact experimental boundary conditions in each case is considered outside the scope of this paper, which focuses on whether the mechanism of grain boundary shears can explain the experimentally observed spatial distribution of the intragranular orientation spread.

\section{X-ray experiment and reconstruction of space filling grain maps}

The material was a fully recrystallised interstitial-free (IF) steel $(99.6 \% \mathrm{Fe})$ with a strong bcc rolling texture. The sample preparation and deformation history, as well as details on the 3DXRD experiment performed at the European Synchrotron Radiation Facility beamline ID11, have been reported previously (Oddershede et al., 2015). In summary both a far-field and a near-field detector was employed to obtain good orientation and spatial resolution, respectively. Details on the analysis of the far-field data and the range of orientations developing within three individual bulk grains of similar orientations were given in the before mentioned paper. The present paper concentrates on analysis of 
the 3D space-filling grain orientation maps reconstructed from the near-field data as described below. Analysis of the far-field data is the basis for this reconstruction, and the relevant far-field information is repeated here.

The sample was tensile deformed ex situ to 3\%, 6\% and $9 \%$. After $9 \%$ deformation the sample was mechanically thinned down to reduce the sample cross section and thereby reduce peak overlap on the detectors. The focus is on the data collected in the undeformed state and on the thinned sample after $9 \%$ tensile deformation. The diffraction data were collected in a layer-by-layer manner with a beam vertically focused to $10 \mu \mathrm{m}$ and horizontally confined by slits to $1.5 \mathrm{~mm}$. The sample displacement between layers was $10 \mu \mathrm{m}$ in the undeformed and $20 \mu \mathrm{m}$ in the deformed case.

\subsection{Undeformed}

In the undeformed state a Frelon4M detector (Labiche et al., 2007) with $2048 \times 2048$ pixels of $50 \times 50 \mu \mathrm{m}^{2}$ was used in the far-field, and from these data grains were indexed by means of GrainSpotter (Schmidt, 2014). The grains were then employed to seed the reconstruction of the 3D space-filling orientation map on the near-field detector, a Quantix with $1536 \times 1024$ pixels of $4.3 \times 4.3 \mu \mathrm{m}^{2}$, using GrainSweeper.3D, a 3D generalization of GrainSweeper (Schmidt et al., 2008). For each $2 \times 2 \times 2 \mu \mathrm{m}^{3}$ voxel in the $840 \times 840 \times 20 \mu \mathrm{m}^{3}$ reconstruction centred around the $700 \times 700 \times 10 \mu \mathrm{m}^{3}$ illuminated volume, the seed grain orientation with highest completeness (defined as the ratio between the number of observed and expected reflections) was assigned. 50 consecutive partly overlapping $20 \mu \mathrm{m}$ reconstructions with a $10 \mu \mathrm{m}$ inter-layer spacing were then stacked along the tensile $z$-axis in order to obtain the 3D space-filling grain map shown in Figure 1(a). For each voxel in the overlapping regions, the orientation with highest completeness was assigned, and voxels with a completeness less than $60 \%$ were eliminated from the map. The undeformed orientation map was then registered to a grain map by assigning adjacent voxels with misorientations less than $1^{\circ}$ to the same grain. This accounts for experimental uncertainties of the order $0.1^{\circ}$ as well as orientation gradients between the 2D slices through the 3D grains of a similar order of magnitude.

The present study focuses on two grains, termed A and B, which lie in the same layers of the sample. A third grain in another part of the sample was also included in the previous paper, but is omitted here to reduce the number of figures. The two selected grains are of representative sizes and shapes as illustrated in Figure 1(b) and (c). Their initial orientations are also similar and close to $<522>$ as shown in Figure 1 (d). The circles mark their final orientations. It is seen that the rotation paths of the two grains are quite different in spite of their initial similarities; grain A shows very small rotation towards the $\langle 100\rangle$ $<111>$ line of the triangle, while grain B rotates substantially towards the <100>$<110\rangle$ line. In fact they represent two extremes when considering all other grains of that orientation in the sample. Figure 1(e) illustrates the positions of the two grains in a 2D slice through the undeformed map. 


\section{2. $9 \%$ deformed}

The reconstruction of the grain orientation maps after $9 \%$ deformation was also performed using GrainSweeper.3D. This time the list of seed orientations was expanded from the roughly 400 per layer used in the undeformed case to almost 3 million. This increased the runtime for a layer from a few minutes to half a day. For the grains of interest, grains $A$ and B, the previously determined intragranular orientation spread approximately matching the far-field data (Oddershede et al., 2015) were employed. In addition all remaining grain orientations indexed in a $150 \mu \mathrm{m}$ slice of the undeformed sample along the $z$-axis embedding grains A and B were added. Centred at each of these undeformed grain orientations an orientation distribution spanning $\pm 10^{\circ}$ in $11^{3}$ steps was added. Finally the grain orientations indexed by GrainSpotter from the $9 \%$ deformed far-field data were added to the seeds for GrainSweeper.3D.

The orientation maps at $9 \%$ deformation were then registered into grains by using a $4^{\circ}$ misorientation criterion, see Figure 1(f) for a slice through the sample after $9 \%$ deformation. Intragranular orientation distributions are present in most grains, though these are not necessarily discernible with the chosen colour code. Recall that the sample was thinned down to reduce peak overlap between the 9\% deformation and the 3DXRD experiment, hence the difference in size of the two corresponding sample cross sections in Figure 1(e) and (f). It is important to note that thinning was done after deformation, i.e. grains $\mathrm{A}$ and $\mathrm{B}$ were bulk grains during the deformation. Three slices separated by approximately $20 \mu \mathrm{m}$ through grains A and B are selected for this study as illustrated in Figure 1(b) and (c). Due to the sampling of layers and the inherent elongation of the sample during tension, the slices in the undeformed and deformed maps could only be matched up within a few micrometres.

\section{Analysis of grain maps}

\subsection{Orientation spread}

The full orientation spreads of grains A and B are illustrated in $<111>$ pole figures in Figure 2(a) and (b). It is seen that the full orientations - and not only the direction of the tensile axes - are very similar for the two grains. The orientation spread within the two grains also share a common directionality as all the spots spread out horizontally in the pole figures. The vertical spread in grain $B$ is somewhat larger than in grain $A$, which corresponds to the larger orientation spread of also the tensile axis in Figure 2(c).

The colour coding in Figure 2 is defined by the extension of spread of the tensile axis into and out of the triangle in Figure 2(c). The orientations furthest into the triangle are coloured red whereas those furthest outside are blue. The same colour coding is employed for the spatially resolved orientation distributions in the deformed maps in subsequent sections (Figure 7 and Figure 8). 


\subsection{Neighbour analysis}

The orientations of all the neighbours to grains $A$ and $B$ are illustrated in the pole figures of Figure 3(a) and (b). These pole figures clearly reveal that both grains $\mathrm{A}$ and $\mathrm{B}$ are situated in local environments that represent the overall strong bcc rolling texture in Figure 3(c) very well. As a quantitative parameter for comparison of the local environment, the mean misorientation between a grain and its immediate neighbours has been suggested (Saxena et al., 2015). This parameter is $39^{\circ}$ for grain $\mathrm{A}$ and $41^{\circ}$ for $\mathrm{B}$. The different behaviours of grains $\mathrm{A}$ and $\mathrm{B}$ can therefore hardly be attributed to the orientation of the neighbouring grains alone, but must also be affected by how these neighbours are connected to the two grains.

Information about grain boundary planes was extracted from the reconstructed grain map of the undeformed sample. The grain boundary plane between grain A (or B) and each neighbouring grain was defined as the best plane through all of the voxels in the neighbour that touch grain A (or B) (Juul et al., 2016). A visual inspection of the thus defined boundary planes was performed, and in general the fitted plane gave a good description of the boundary as exemplified in Figure 4(a). In a few cases an alternative description was adopted, especially for boundaries with very small areas (less than 50 voxels). Figure 4(b) shows an example of a poor fit using a single boundary plane. A much better fit to the voxels of this boundary was obtained by describing the boundary as two different planes depending on the position along the z-axis. The improved boundary planes are illustrated in Figure 4(c). Thus equipped with the orientations of grain boundary plane normals, as well as the volume-weighted mean orientations of grains A, B and their respective neighbours in the undeformed sample, all of the input needed for the ALAMEL modelling has been extracted from the 3DXRD experiment.

\section{Model implementation}

The ALAMEL model considers the localized interaction between two grains in a region close to the boundary between them. This boundary is assumed to be planar. The boundary region modelled has an unspecified extension into both grains as indicated by the grey circle in Figure 5(a). The basic assumption of the ALAMEL model is that the interaction between the two grains is accommodated by co-operative grain boundary shears. With a boundary coordinate system defined with the boundary normal as the 3-direction as in Figure 5, three types of co-operative shear are possible. Following the suggestion of (Van Houtte et al., 2005) only shears on the boundary plane in the 1- and 2-directions, termed $\gamma_{13}$ and $\gamma_{23}$, as illustrated in Figure 5(b)-(c), are employed. The two shears are cooperative in the sense that they are of equal magnitude in the two grains but of opposite sign. Inclusion of the third type of co-operative shear, i.e. on the 1-plane in the 2-direction (or vice versa), requires shears of the same sign in the two grains in order to avoid grain boundary sliding. Introduction of these shears of the same sign would, however, lead to a skewed distortion of the grain boundary plane, which may be unphysical, although an invariant method for selection of 
the coordinate system in the grain boundary for introduction of co-operative $\gamma_{12}$ or $\gamma_{21}$ shears has been devised (Mánik and Holmedal, 2013).

Following (Van Houtte et al., 2005), the practical implementation of the cooperative grain boundary shears consists of extending the standard equations in the Taylor model accounting for slip on the crystallographic slip systems with pseudo slip systems describing the boundary shears. The boundary plane normal (3 in Figure 5) is the slip plane normal of these pseudo slip systems, and the slip direction is the 1- or 2-direction. The pseudo slip systems in the two interacting grains are identical, but must have activities of equal magnitudes and opposite signs.

The strain tensors $\boldsymbol{\varepsilon}_{\boldsymbol{A} / \boldsymbol{B}}$ for grain A (or B), and $\boldsymbol{\varepsilon}_{\boldsymbol{N} \boldsymbol{B}}$ for the neighbouring grain are defined in the standard way by the Schmid factor, $\boldsymbol{m}_{i}^{\boldsymbol{A} / \boldsymbol{B}}$ or $\boldsymbol{m}_{\boldsymbol{i}}^{N \boldsymbol{B}}$, of and the shear, $\gamma_{i}^{A / B}$ or $\gamma_{i}^{N B}$, on the crystallographic slip systems. The Schmid factor of the pseudo slip systems is calculated in complete analogy with the crystallographic case, i.e. as

$$
\boldsymbol{m}_{\boldsymbol{i 3}}=\operatorname{sym}\left\{e_{i} \otimes e_{3}\right\}
$$

where $e_{i}$ is a unit vector along the i-direction of the boundary coordinate system $(1,2,3)$, expressed in the loading coordinate system $(x, y, z)$ as also the other terms in the basic equations of the model:

$$
\begin{gathered}
\boldsymbol{\varepsilon}_{\boldsymbol{A} / \boldsymbol{B}}=\sum_{\text {cryst }} \gamma_{i}^{A / B} \boldsymbol{m}_{\boldsymbol{i}}^{\boldsymbol{A} / \boldsymbol{B}}+\sum_{\text {pseudo }} \gamma_{i 3}^{A / B} \boldsymbol{m}_{\boldsymbol{i} \mathbf{3}} \\
\boldsymbol{\varepsilon}_{\boldsymbol{N} \boldsymbol{B}}=\sum_{\text {cryst }} \gamma_{i}^{N B} \boldsymbol{m}_{\boldsymbol{i}}^{\boldsymbol{N} B}+\sum_{\text {pseudo }} \gamma_{i 3}^{N B} \boldsymbol{m}_{\boldsymbol{i} 3} \\
\sum_{\text {pseudo }}\left(\gamma_{i 3}^{A / B}-\gamma_{i 3}^{N B}\right) \boldsymbol{m}_{\boldsymbol{i}}=0
\end{gathered}
$$

A prescribed strain tensor , $\boldsymbol{\varepsilon}_{\boldsymbol{r} \boldsymbol{} \boldsymbol{g}}$ (also defined in the loading coordinate system $(x, y, z)$ is enforced on the boundary region consisting of equal volumes of the two interacting grains. While the boundary region on average deforms with the prescribed strain, the introduction of shears in the boundary coordinate system $(1,2,3)$ leads to strain partitioning between grain A (or B) and the neighbouring grain.

As in the Taylor model, the equations are solved for the unknown values of $\gamma_{i}^{A / B}$, $\gamma_{i}^{N B}$ and $\gamma_{i 3}^{A / B}\left(=-\gamma_{i}^{N B}\right)$ with the constraint that the plastic work, $\mathrm{W}$, to which only the crystallographic slip systems contribute, is minimised:

$$
W=\tau_{\text {crss }}\left(\sum_{\text {cryst }} \gamma_{i}^{A / B}+\sum_{\text {cryst }} \gamma_{i}^{N B}\right)
$$


assuming equal critical resolved shear stresses, $\tau_{\text {crss }}$, on all crystallographic slip systems. For the present bcc material, crystallographic slip systems of the $\{110\}<111>$ family are considered. In between simulation steps, the lattice orientations of the two interacting grains as well as the orientation of the boundary plane are updated by their rigid body rotations. Rate independence is assumed and hardening is neglected.

It is inherent to the model that it does not account for the accommodation of the resulting plastic heterogeneities in the grain interior. Consequently, the present study focuses on the orientations developing in the immediate vicinity of the boundaries. When modelling the intragranular orientation spread of grain A (or B) in the present case, each boundary region is modelled independently and contributes a single orientation to the orientation spread.

\subsection{Boundary conditions}

While the ALAMEL model is designed for strain variations within the boundary region, the boundary conditions in terms of a strain tensor enforced on the entire grain boundary region, $\boldsymbol{\varepsilon}_{\boldsymbol{r e g}}$, must be prescribed. When applying the model to a statistical ensemble of grain pairs, the enforced strain tensor is usually taken to be identical to that expected for the entire sample. The classical Taylor model predicts a Lankford parameter $\left(\mathrm{R}=\varepsilon_{x x} / \varepsilon_{y y}\right)$ of 3 based on the measured set of grain orientations in the sample (see Figure 3(c)). This is as expected for tension along the original rolling direction in view of the strong bcc rolling texture of the initial material.

It is, however, well-known from direct experimental evidence obtained by grain shape variations (Wu et al., 2005), digital image correlation (Badulescu et al., 2011; Martin et al., 2014) or studies of intragranular orientation spreads (AllainBonasso et al., 2012; Obstalecki et al., 2014) - often supported by crystal plasticity simulations - that substantial plastic heterogeneities occur on scales in between the size of the sample and a localised grain boundary region. These include both variations in the magnitude of the strain, the Lankford parameter and the principal strain directions (i.e. introduction of shear components absent in the macroscopic loading coordinates of the sample).

Although finite element-based simulations may be employed to estimate the average strain of the grain boundary region, $\boldsymbol{\varepsilon}_{\boldsymbol{r} \boldsymbol{e g}}$, to be enforced by the ALAMEL model, the present choice is to roughly estimate this strain and the effect of varying it on the ALAMEL predictions. By trial-and-error, suitable boundary conditions, $\boldsymbol{\varepsilon}_{\boldsymbol{r e g}}$, are identified. Optimisation of the boundary conditions for each grain boundary region to obtain exact agreement with experiment was not pursued because a range of conditions give fairly similar results as illustrated in the next section. Instead a common method giving good qualitative and near quantitative agreement for all boundary regions was pursued.

As a first approach the macroscopic strain of the sample, i.e. $9 \%$ tensile elongation, $\varepsilon_{z z \text { reg }}$, and a Lankford parameter, $R_{\text {reg }}$, of 3 , was enforced on all grain boundary regions, i.e. 


$$
\boldsymbol{\varepsilon}_{\text {reg }}=\varepsilon_{z z r e g}\left(\begin{array}{ccc}
\frac{-R_{r e g}}{R_{r e g}+1} & 0 & 0 \\
0 & \frac{-1}{R_{r e g}+1} & 0 \\
0 & 0 & 1
\end{array}\right)
$$

Secondly, both the tensile elongation and $\mathrm{R}_{\text {reg }}$ were varied and finally $\mathrm{R}_{\mathrm{reg}}$ was optimised for each individual boundary region to give the smallest work as determined from Eq. (3). For simplicity, only $\varepsilon_{z z \text { reg }}$ and $R_{r e g}$ in $\boldsymbol{\varepsilon}_{\text {reg }}$ are varied, while the shear components are assumed vanishing. However, when the model partitions $\varepsilon_{r e g}$ between the two interacting grains, the strains, $\varepsilon_{A / B}$ and $\varepsilon_{N B}$, in general contain shear components. None of the simulations put restrictions on the strain partitioning between the two interacting grains or on the shears along the boundary.

\section{Modelling results}

\subsection{Overall orientation spread}

In order to best compare the predicted orientation spreads to the experimentally observed spreads, both the spread of the tensile axis and the spread of one of the $<111>$ poles are presented. The $<111>$ pole chosen lies far from the tensile axis and thus presents the spread of a direction, which differs significantly from the tensile axis. Figure 6 presents stereographic triangles of the tensile axis and the $<111>$ pole as insets for each simulation. The experimental orientation spread is repeated from Figure 2. For reference, the initial orientation of the grain is included as a green star. The black dots are the orientations predicted by the ALAMEL model for the boundary regions. Figure 6(a)-(d) and Figure 6(e)-(f) show the predictions for grains A and B, respectively. For each grain, the boundary conditions, i.e. $\boldsymbol{\varepsilon}_{\boldsymbol{r e g}}$, were varied as described in section 4.1, and some representative examples are presented.

Figure 6(a) shows the result of applying the macroscopic strain conditions with $9 \%$ tensile elongation and $R_{\text {reg }}=3$ to grain A. Except for a single case indicated by an arrow in Figure 6(a), all rotations of the tensile axis are in the direction observed experimentally. It is, however, evident that the rotations are overestimated. Variations of the Lankford parameter with $9 \%$ tensile elongation did not yield better predictions. As seen in Figure 6(b), reduction of the tensile strain to $4.5 \%$ with $R_{r e g}=3$ gave much better agreement. The magnitudes of the predicted rotations and orientation spread are now closer to the experiment. Figure 6(c) presents additional predictions with an enforced elongation of $4.5 \%$ but where $R_{\text {reg }}$ has been allowed to vary in the range 1 to 8 to minimise the work for each individual boundary region. For all practical purposes this is similar to Figure $6(\mathrm{~b})$, and the mean of the $R_{\text {reg }}$ values giving the smallest work for each region is 3.1. The range of these $R_{\text {reg }}$ values spans from 1 to 5.7.

Enforcement of an elongation of $4.5 \%$ for grain $\mathrm{A}$ is a substantial reduction compared to the nominal $9 \%$ elongation of the sample. Although a factor of two 
in the major strain component is not outside the bounds found in some simulations (Nicaise et al., 2011) and experimentally in large columnar grains (Badulescu et al., 2011), it is large compared to other simulations which indicate levels of the order of 20-35\% (Li et al., 2008; Sarma and Dawson, 1996). To investigate the sensitivity of the predictions to the choice of boundary conditions, simulations with intermediate tensile strains were also conducted. It was found that the combination of enforcing an intermediate elongation and increasing $R_{\text {reg }}$ also produces orientation changes in grain $\mathrm{A}$, which agree well with the experimental observations. The predictions for a tensile strain of $7 \%$ and an enforced $R_{\text {reg }}$ of 5 for all boundary regions are illustrated in Figure 6(d). The resulting orientation spread is almost the same as in Figure 6(b) and (c). It is, however, noteworthy that the prediction for the grain boundary region which rotates opposite of the experimentally observations as marked by the arrow in Figure 6(a) improves with increasing $R_{\text {reg }}$ (see arrow in Figure 6(d)).

Corresponding predictions for grain B in Figure 6(e) and (f) match the overall experimental rotation angles for an enforced tensile elongation of $9 \%$ for the boundary region. Also with respect to the orientation spread, the agreement is good. The prediction in Figure 6(e) with a fixed $R_{\text {reg }}$ of 3 for all boundary regions deviates only minutely from the one in Figure 6(f), where $R_{\text {reg }}$ is optimised to give the minimum work in each boundary region. In the latter case the mean $R_{\text {reg }}$ of 2.8 is also close to 3 , with a span from 1 to 4 .

The conclusion on the simulations with varying boundary conditions is that the predicted orientation spread is fairly insensitive to the exact boundary conditions employed. This demonstrates that the local orientation evolution at the boundaries is dominated by co-operative boundary shears with a minor influence of the longer range interactions leading to deviations in the average strain of the boundary region. However, quantitative agreement between ALAMEL predictions and experiment cannot be obtained when applying the same boundary conditions to grain $\mathrm{A}$ as to grain B. Good agreement was obtained for grain A using 7\% elongation with $R_{r e g}=5$, and $9 \%$ with $R_{r e g}=3$ for grain $\mathrm{B}$. These are realistic boundary conditions, and although they may not represent the actual experimental situation, they are employed in the predictions of the spatial distribution of the orientations as presented in the next section. Subsequent sections look into the differences between the individual grain boundary regions, which create the orientation spread.

\subsection{Spatial distribution of orientation spread}

Figure 7 and Figure 8 compare the predictions with the experimental data for grains A and B, respectively. For clarity, the predicted orientations at the grain boundaries are presented in the undeformed maps, which contain all the neighbours of grains $\mathrm{A}$ and $\mathrm{B}$. The colour of all neighbouring grains in both deformed ((a)-(c)) and undeformed ((d)-(f)) maps is according to the orientation of their tensile axis as in Figure 1. It is seen that the neighbours change their orientation during the tensile deformation and a developing orientation spread may be discerned within some of these. The colour coding of these grains is, however, not optimised to present such relatively small orientation differences. 
To emphasize the orientation spread within grains A and B the colour coding of these two grains is the same as in Figure 2, i.e. red colours represent rotations into the stereographic triangle whereas blue colours indicate orientation spread in the opposite direction. The experimentally observed spatial distribution of the orientation spread after deformation is presented in Figure 7 and Figure 8 (a)(c) for the three slices of grain A and B. For comparison, the orientations predicted by the ALAMEL model in the grain boundary regions of grains A and $B$ are indicated by coloured lines of arbitrary width in the undeformed maps ((d)(f)), using the same colour coding as in the deformed maps. The lines follow the trace of the determined boundary planes from the undeformed maps.

\subsubsection{Grain A}

For grain A, the predictions in Figure $7(d)$ and (e) show dominance of red colours, i.e. orientation spread extending into the triangle, at the grain boundaries at the high-y part of the grain, which agree well with the experimental results in Figure 7(a) and (b). Also the occurrence of blue colours, i.e. orientation spread in the opposite direction, at the low-y part of grain A is predicted. At the (high- $x$, low $-y$ ) part of grain A the experimentally observed change from blue in (a) to red in (b) is correctly reproduced by the model, (d) to (e), as the result of the change of neighbouring grains in this position.

Comparison of Figure 7(c) with (f) reveals a much poorer agreement, which may be a consequence of taking a slice from the very bottom of grain A as illustrated in Figure 1(b) and discussed in section 6.2.

\subsubsection{Grain B}

Figure 8 compares the model predictions for grain B with the experimental data. In Figure 8(a) the dominant colour of grain B is orange (i.e. rotation into the triangle), but at the extremes along $y$ in the map, the orientation spread is out of the triangle (i.e. blue colours). This is in agreement with the predictions in Figure 8(d). Note that the pink region in the (low- $x$,high- $y$ ) part of Figure 8(a) is a grain lying above grain B in the sample. Its occurrence in only the deformed map is due to the slight mismatch between the layers mapped before and after deformation.

The occurrence of blue boundary regions in the high-y parts of grain B in the deformed maps in Figure 8(b) and (c) is in excellent agreement with the model predictions in Figure 8(e) and (f). In the same figures, the low-y boundary regions of grain $B$ are predicted to have reddish colours, which also matches the experimental map well. The (high- $x$,low-y) boundary in Figure 8(e) is even correctly predicted to have the deepest red colour, i.e. to have the orientation bounding the orientation spread into the triangle.

It is noteworthy that the extreme low- $x$ boundary region in the deformed maps of grain B changes from reddish to blue colours from Figure 8(a) to (b), even though the neighbouring grain in the two slices is the same. As illustrated in Figure 4(c), this boundary was fitted with two different planes, the upper plane valid for Figure 8(a) and the lower for Figure 8(b) and (c). Using these boundary planes, the ALAMEL model correctly reproduces the change of grain orientations in the grain boundary regions as represented by the change of colour from Figure $8(d)$ to $(e)$. 


\subsection{Strain partitioning and rotation angles}

The boundary conditions, $\boldsymbol{\varepsilon}_{\boldsymbol{r} e g}$, enforced on the grain boundary regions are fixed to $7 \%$ elongation and $R_{r e g}=5$ for grain $\mathrm{A}$ and $9 \%$ and $R_{\text {reg }}=3$ for grain $\mathrm{B}$. The ALAMEL model, however, partitions the strain between the two interacting grains. The predicted variations of the tensile strain, $\varepsilon_{z z A / B}$, and the Lankford parameters, $\mathrm{R}_{\mathrm{A} / \mathrm{B}}$, for the various regions within grain $\mathrm{A}$ and $\mathrm{B}$ are presented in Figure 9.

On average the boundary regions of grain $\mathrm{A}$ deform with the enforced tensile strain of 7\%, whereas the mean for grain B is larger than the enforced value (9.9\% vs. $9 \%$ ). The tensile strain is also more narrowly distributed for grain A than for grain B. The width of these distributions may be decreased by inclusion of hardening in the simulations, as the most deforming grain will harden more. The distribution of $\mathrm{R}_{\mathrm{A}}$ is on the other hand wider than for $\mathrm{R}_{\mathrm{B}}$. Both grains have a very large value at a single boundary, i.e. almost plane strain deformation. This makes discussion of the mean values irrelevant. However, the medians of the distributions are 4.5 and 2.5 for grains $\mathrm{A}$ and B, respectively, which are quite close to the enforced $R_{\text {reg }}$ of 5 and 3. The different boundary conditions, $\boldsymbol{\varepsilon}_{\text {reg }}$, for the two grains was motivated in the observed smaller rotation angles for grain $\mathrm{A}$, and grain $\mathrm{A}$ therefore has a much smaller mean rotation angle. The distribution around the mean values is, however, similar for the two grains.

\subsection{Slip system variations}

Analysis of the slip systems predicted to be active by the ALAMEL model reveals that most of the slip occurs on four slip systems, which is also in agreement with the classical Taylor model in uniaxial tension (irrespective of the $R_{r e g}$-value variations employed here). These systems are the primary and conjugate systems (P: $(0 \overline{1} \overline{1})[11 \overline{1}]$ and $C:(\overline{1} 01)[111])$ and two other systems that are codirectional to these (CDP: $(1 \overline{1} 0)[11 \overline{1}]$ and CDC: $(1 \overline{1} 0)[\overline{111}])$. All of these are also among the systems with high Schmid factors.

When averaging over all grain boundary regions, the combined activities of the $\mathrm{P}$, C, CDP and CDC systems as predicted by the ALAMEL model account for 90 and $95 \%$ of the slip in the grain boundary regions of grains A and B, respectively. The mean activity on the primary system accounts for 38 and $41 \%$. The corresponding numbers for the conjugate system are 33 and $22 \%$. The fact that primary and conjugate slip is on average more balanced in grain A while the primary slip system is almost twice as active as the conjugate in grain $B$ is in agreement with the larger orientation spread of the tensile axis for grain A out of the stereographic triangle in Figure 6(c). The two slip systems have equal Schmid factors at the $<100>-<111>$ symmetry line of the triangle and they cause rotations in nearly opposite directions.

Considering the slip distributions for the individual regions in both grains, the variations are quite large. Figure 10 presents the slip distribution for each boundary region in the form of a pie diagram. For clarity only the central layer from each grain (i.e. the maps from Figure 7(e) and Figure 8(e)) is included. Each slice in the pie diagram represents the relative activity of a slip system, summed over the entire simulation from the undeformed to the deformed state (enforcing 
the same conditions onto each region as in Figure 7 to Figure 9). Red and blue slices account for primary and conjugate slip, respectively. This colour selection matches the colour coding of the intragranular orientation spread as primary slip causes the tensile axis to rotate into the triangle while conjugate slip gives rotations out of the triangle. The two grey shades are the CDP and CDC systems and other systems are marked by black.

For grain A in Figure 10(a) there is good agreement between the orientations and the slip distributions at the grain boundaries. In the grain boundary regions at the extremes along $y$ of grain $A$, which develop orientations that are represented by red colours, the primary slip system is substantially more active than the conjugate. At the extremes along $x$ of grain A the primary and conjugate slip systems are equally active, and orientations represented by orange/yellow colours evolve. At the only boundary of grain A in Figure 10(a) with dominance of conjugate slip in the (low- $x$,low- $y$ ) corner, the orientation at the boundary is at the blue end of the colour scale and primary slip is about half of the conjugate slip fraction.

In grain $\mathrm{B}$, the tendency for dominance of primary slip in boundary regions predicted to rotate furthest into the triangle and therefore represented by red colours also holds at the low-y part in Figure 10(b). However, in this case the fractions of primary and conjugate slip predicted are almost the same even though the resulting orientations are mapped onto colours spanning from orange to deep red. Prediction of blue colours along the high-y boundaries of grain B is also in agreement with large fractions of conjugate slip. However, the conjugate slip only significantly exceeds the primary at a single boundary in the high-y part of grain B. It is noteworthy that this boundary region is the one developing the deepest blue colours in the experimental map of Figure 8(b) although the predicted orientations give a lighter shade of blue.

The discrepancies between slip distributions and colour matching of orientations for grain B compared to A is an artefact of the range of the red-blue scale being different for grains $\mathrm{A}$ and $\mathrm{B}$. The colour coding is fixed to the magnitude of the experimental lateral orientation spread of the tensile axis for each grain. As also noted above and seen in Figure 6(c), the orientation spread of grain A extends much further out of the triangle than for grain $B$, meaning that the darkest blue shade corresponds to quite different orientations with respect to the $<100>-<111>$ symmetry line in the two grains. For this reason, almost balanced primary and conjugate slip leads to orientations represented by orange/yellow in grain $\mathrm{A}$, but light blue for grain $\mathrm{B}$.

\section{Discussion}

\subsection{Accuracy of experimental grain maps}

As described in section 2.2 the reconstruction of the spatial orientation distribution in grains $\mathrm{A}$ and $\mathrm{B}$ from the near-field 3DXRD data in the $9 \%$ deformed sample was done assuming a broad, yet discrete orientation distribution centred around orientations previously found to approximate the 
peak spread of selected diffraction spots on the far-field detector (Oddershede et al., 2015). In order to demonstrate that the orientations determined in the reconstructed deformed grain maps are more precise than our previous approximation, forward modelling of the diffraction spots was employed (Wong et al., 2013). The forward modelling approach simulates the spots on the far-field detector based on the orientations in the deformed map. This method has previously been applied in combination with crystal plasticity to study slip activity (Pagan and Miller, 2014) and cyclic loading (Obstalecki et al., 2014; Wong et al., 2015). The spatial distribution of orientation and strain within a grain, as well as experimental features such as the X-ray energy bandwidth and the spatial distortion of the detector are accounted for in the forward modelling.

One such example of three selected diffraction spots is shown in Figure 11. In the left column the experimental far-field diffraction spots after $9 \%$ deformation are shown for a slice through grain A, while the diffraction spots in the central column were forward projected using our previous approximate orientation distribution, and to the right the same diffraction spots have been forward projected from the spatially resolved orientation distribution modelled on the near-field data. Both sets of forward projected spots seem a bit too narrow compared to the experimental counterparts, possibly because the modelled detector point spread of 3 pixels is underestimated. However, of the two distributions the near-field results to the right show the best correspondence with the experimental far-field peak spreads to the left.

The grain maps were reconstructed with a smaller voxel size of $2 \times 2 \times 2 \mu \mathrm{m}^{3}$ than perhaps warranted by the pixel size of the near-field detector of $4.3 \times 4.3 \mu \mathrm{m}^{2}$. The finer resolution was chosen to produce a smoother map, while the determination of the grain boundary planes was performed based on a binned map with a resolution of $4 \times 4 \times 4 \mu \mathrm{m}^{3}$. All comparisons between experimental and predicted orientations are based on fairly large numbers of voxels along the grain boundaries and are therefore not affected by the exact spatial resolution.

In order to extract representative grain boundary planes as input to the ALAMEL model, the experimental three-dimensional grain structure was approximated by facetted grains using fitted planar grain boundaries. In a few cases this was deemed insufficient and two different planes were employed to give a much more realistic grain structure (see Figure 4). More detailed representation of the grain boundaries, e.g. in the form of the voxelised field of grain boundary normals (Liberman et al., 2015), might have been employed, but would not be compatible with the present analysis of grain interactions in the simplest possible model framework.

\subsection{Limitations of the ALAMEL model}

The ALAMEL model considers the local interaction by grain boundary shear between two grains connected by a planar boundary and was originally devised as a statistical model. The present study applies it to specific grains to evaluate the importance and effectiveness of grain boundary shear as an isolated grain 
interaction mechanism, finding that such shears are important as they at least qualitatively explain both the directionality of the intragranular orientation spread and its spatial distribution.

The relative simplicity and microstructural assumptions in the ALAMEL model obviously gives rise to some limitations in its application to specific grains compared to more computationally complex finite-element based crystal plasticity models. The problem of identifying the average strain, $\varepsilon_{r e g}$, of the grain boundary region, which is an inherent part of finite-element based simulations, has already been treated in detail in the previous sections and probably constitutes the major difficulty in obtaining quantitative predictions with the ALAMEL model.

Another limitation of the ALAMEL model compared to finite-element methods is that each boundary region is modelled independently, i.e. only the interaction between two grains is considered. This is also the reason why the ALAMEL model does not specifically account for the spatial extension of the grain boundary region or the orientations in the centre of the grain, although a geometrical method to estimate these has been proposed (Van Houtte et al., 2005). The present results indicate that this assumption of independency is merited in most cases. However, the model clearly broke down for the very bottom part of grain $\mathrm{A}$, which has a small cross section and where the interaction with grains lying below grain A must also expected.

Finally, the assumption of planar grain boundaries is a simplification of the real microstructure. Appropriate meshing in finite-element based models can yield much more accurate representations. The present study employed piece-wise planar boundaries where the assumption of planarity was clearly inappropriate. To test the general sensitivity to the grain boundary plane, the ALAMEL predictions for grains $A$ and $B$ were repeated with each boundary plane rotated $\pm 5^{\circ}$ around the $x-, y$ - and $z$-axes of the tensile sample. On average this caused deviations of the final predicted orientations at the grain boundary regions of grains $A$ and $B$ of $1.0^{\circ}$ and $1.6^{\circ}$, respectively. These uncertainties are significantly smaller than the largest internal misorientation within grains $\mathrm{A}$ and $\mathrm{B}$ of $5^{\circ}$ and $6^{\circ}$, and therefore do not affect the qualitative conclusions of the present study.

Another recognised problem with the ALAMEL model is that shears at the grain boundaries are free-of-work. If the grain boundary coordinate system coincides with a most stressed sample plane or slip system, unrealistically large grain boundary shears may dominate the model prediction. The fraction of boundary shear in a grain may be defined as

$$
\left(\left|\gamma_{13}\right|+\left|\gamma_{23}\right|\right) /\left(\left|\gamma_{13}\right|+\left|\gamma_{23}\right|+\sum_{i}\left|\gamma_{i}^{A / B}\right|\right)
$$

where $\gamma_{i}$ is the shear on the slip systems and $\gamma_{13}$ and $\gamma_{23}$ the shears in the grain boundary. On average this fraction is 0.17 and 0.22 for grains A and B, respectively. The maximum shear fraction is about 0.33 and occurs for one grain boundary region of grain A and two in grain B. In none of these boundary regions the boundary plane aligns closely with either a macroscopically most stressed 
plane, inclined $45^{\circ}$ to the tensile axis, or with a slip plane. The shear predicted by the ALAMEL model at the grain boundaries investigated here are therefore not physically unrealistic artefacts introduced by geometry. These calculations further show that the modelled grain boundary shears are substantial and their introduction an important modification to the standard Taylor model.

In spite of its limitations, the qualitative success of the present ALAMEL predictions confirms the physical realism of the basic assumptions of the ALAMEL model, and in particular that co-operative grain boundary shears are an important grain interaction mechanism. At the expense of more complex calculations, finite-element based models are obviously capable of providing more detailed predictions of the local strain conditions and the orientations in the grain interior. Quantitative predictions by finite element-based crystal plasticity for the two grains considered here will be pursued in a future study.

\subsection{Grain interaction mechanisms and slip systems}

Interaction between plastically hard and soft grains is another suggested mode of grain interaction, where softer grains are expected to deform more than hard ones, e.g. (Raabe et al., 2001; Vachhani et al., 2016). The ALAMEL model implicitly accounts for this interaction by partitioning the strain enforced on the entire grain boundary region between the two grains. This partitioning is illustrated in the strain distributions in Figure 9. The ratio of the Taylor factors of the interacting grains is often employed as a measure of the expected strain partitioning. Using $\varepsilon_{z z}=7 \%$ and $R_{r e g}=5$ for grain A and with $\varepsilon_{z z}=9 \%$ and $R_{r e g}=3$ for grain $B$, the Taylor factors of the two grains are 2.75 and 3.15, respectively. This is in disagreement with the present finding that grain B must deform more. The Taylor factor of grain A lies within $70-101 \%$ of the Taylor factors for its neighbouring grains. The Taylor factor of grain B falls between 86 and $116 \%$ of its neighbours. Grain A is therefore estimated to be plastically softer than or comparable to its neighbours, whereas grain B has both somewhat softer and harder neighbours in roughly equal numbers. This may be the origin of the wider distribution of the predicted tensile strains in grain B (Figure $9(d)$ ) compared to grain A in (Figure 9(a)). There is, however, no direct correlation between the ratios of Taylor factors and predicted tensile strains in the grain boundary regions.

Most of the slip activities are concentrated on the primary and/or conjugate slip systems, which have the highest Schmid factors. Slip transmission through grain boundaries is often observed in localised form (Britton and Wilkinson, 2012). Although this spatially localised phenomenon is beyond the resolution of the present grain maps, potential correlations between the preferred activation of the primary and/or conjugate slip system and the slip systems in the neighbouring grain were investigated. Several criteria for slip transmission have been devised (Bayerschen et al., 2016). The alignment of the slip systems in the two interacting grains is a main component in most of these, parametrised by the angle between the two intersection lines between the boundary plane and the slip planes. For the present grains, the alignment of slip planes is in general not favourable for slip transmission. In the few cases where the angle between 
intersection lines is of the order of $10^{\circ}$ for either the primary or conjugate slip system, this system was not the preferred one in the grain boundary region.

The grain interaction predicted by the ALAMEL model is not strong enough to cause significant activation of other slip systems than those expected based on a classical Taylor prediction. The grain interaction, however, greatly affects the relative activities of these systems as demonstrated in Figure 10. The finding of large intragranular variations in the activities of the most active systems is also in agreement with simulations of the orientation spread of split aluminium samples subjected to plane strain compression (Quey et al., 2015). Intergranular differences in the rotation paths of similarly oriented grains are also in agreement with intergranular variations in the relative activities of such systems (Winther, 2008).

The operation of the same set of slip systems throughout the grain, although with different relative activities, is also in agreement with general observations of a grain orientation (Cizek et al., 2005; Huang and Winther, 2007; Le et al., 2012; S. Wang et al., 2014) and slip system dependence (Winther and Huang, 2007) of the alignment of extended planar dislocation boundaries within a grain, even to small grain sizes (Le et al., 2013). Also the spacing and misorientation across these boundaries exhibit grain orientation dependence (Hansen et al., 2006). Recent analyses of selected boundaries have revealed that these contain dislocations from the active slip systems (Hong et al., 2013; McCabe et al., 2004; Wei et al., 2011), which assemble in a low-energy-dislocation-structure (LEDS), ideally free of long-range elastic stresses (McCabe et al., 2004; Wei et al., 2011; Winther et al., 2015). Typically two sets of intersecting dislocation boundaries evolve and are observed throughout the entire grain, suggesting the same set of operating systems everywhere. The spacing and overall clarity of these, however, often varies within the grain, in agreement with the existence of local variations in the activities of the operating systems. Like the evolving deformation texture, the preferred alignment of dislocation boundaries contributes to the mechanical anisotropy (Beyerlein and Tome, 2007; Winther, 2005).

\section{Conclusions}

The deformation-induced intragranular orientation spread has been analysed from 3DXRD data in two grains of similar orientation, size and shape embedded in three-dimensional environments of neighbouring grains, which are also of similar orientations and representative of the bulk texture. The two grains are deliberately selected among a number of grains with similar orientation because they exhibit the extreme differences in average rotations.

- The differences in absolute rotation angles between the two grains can only be explained by longer range grain interactions, which result in different average strains of the grains.

- A fairly large range of realistic strain conditions for both of the investigated grains yields qualitatively successful ALAMEL model predictions with respect 
to the directionality and magnitude of the intragranular orientation spread and also the spatial distribution of the orientations along the grain boundaries. The qualitative success and robustness of the ALAMEL model demonstrate that co-operative shear at the grain boundaries is a significant grain interaction mechanism.

- Most of the orientation spread is caused by relative variations in the activities of the primary and conjugate slip systems with high Schmid factors.

Although limited to two similar grains in similar neighbourhoods, the findings confirm the general success of the ALAMEL model in prediction of bulk textures, as well as the need for detailed microstructural knowledge to predict the behaviour of individual grains.

\section{Acknowledgements}

The authors are grateful to Drs. Lawrence Margulies, Xiaoxu Huang and Henning F. Poulsen for participating in the synchrotron experiment. Drs. Oddershede and Winther acknowledge support from the Danish Independent Research Council | Technology and Production Sciences grant no. DFF - 1335-00220 within which parts of the project have been carried out. The Danish Agency for Science, Technology and Innovation is acknowledged for covering the expenses in relation to the synchrotron experiment (through Danscatt). The European Synchrotron Radiation Facility is acknowledged for granting beamtime for the experiment.

\section{References}

Abdolvand, H., Majkut, M., Oddershede, J., Schmidt, S., Lienert, U., Diak, B.J., Withers, P.J., Daymond, M.R., 2015a. On the deformation twinning of $\mathrm{Mg}$ AZ31B: A three-dimensional synchrotron X-ray diffraction experiment and crystal plasticity finite element model. Int. J. Plast. 70, 77-97. doi:10.1016/j.ijplas.2015.03.001

Abdolvand, H., Majkut, M., Oddershede, J., Wright, J.P., Daymond, M.R., 2015b. Study of 3-D stress development in parent and twin pairs of a hexagonal close-packed polycrystal: Part I - in-situ three-dimensional synchrotron Xray diffraction measurement. Acta Mater. 93, 246-255. doi:10.1016/j.actamat.2015.04.020

Afrin, N., Quadir, M.Z., Ferry, M., 2013. On the Complexities of Microband Interactions in a Cold-Deformed Goss-Oriented Ni Single Crystal. Metall. Mater. Trans. B 45, 345-351. doi:10.1007/s11663-013-9827-7

Allain-Bonasso, N., Wagner, F., Berbenni, S., Field, D., 2012. A Study of the heterogeneity of plastic deformation in IF steel by EBSD. Mater. Sci. Eng. A 548, 56-63. doi:10.1016/j.msea.2012.03.068

Aydiner, C.C., Bernier, J. V., Clausen, B., Lienert, U., Tomé, C.N., Brown, D.W., 2009. Evolution of stress in individual grains and twins in a magnesium alloy aggregate. Phys. Rev. B - Condens. Matter Mater. Phys. 80, 1-6.

doi:10.1103/PhysRevB.80.024113 
Badulescu, C., Grédiac, M., Haddadi, H., Mathias, J.D., Balandraud, X., Tran, H.S., 2011. Applying the grid method and infrared thermography to investigate plastic deformation in aluminium multicrystal. Mech. Mater. 43, 36-53. doi:10.1016/j.mechmat.2010.11.001

Barton, N.R., Bernier, J. V., 2012. A method for intragranular orientation and lattice strain distribution determination. J. Appl. Crystallogr. 45, 1145-1155. doi:10.1107/S0021889812040782

Bayerschen, E., McBride, A.T., Reddy, B.D., Böhlke, T., 2016. Review on slip transmission criteria in experiments and crystal plasticity models. J. Mater. Sci. 51, 2243-2258. doi:10.1007/s10853-015-9553-4

Bernier, J. V., Barton, N.R., Lienert, U., Miller, M.P., 2011. Far-field high-energy diffraction microscopy: a tool for intergranular orientation and strain analysis. J. Strain Anal. Eng. Des. 46, 527-547. doi:10.1177/0309324711405761

Beyerlein, I.J., Tome, C.N., 2007. Modeling transients in the mechanical response of copper due to strain path changes. Int. J. Plast. 23, 640-664.

Bhattachayya, A., El-Danaf, E., Kalidindi, S.R., Doherty, R.D., 2001. Evolution of grain-scale microstructure during large strain simple compression of polyrystalline aluminum with quasi-columnar grains: OIM measurements and numerical simulations. Int. J. Plast. 17, 861.

Bieler, T.R., Wang, L., Beaudoin, A.J., Kenesei, P., Lienert, U., 2014. In Situ Characterization of Twin Nucleation in Pure Ti Using 3D-XRD. Metall. Mater. Trans. A 45A, 109-122. doi:10.1007/s11661-013-2082-3

Bishop, J., Hill, R., 1951. A theory of the plastic distortion of a polycrystalline aggregate under combined stresses. Philos. Mag. 42, 414-427.

Borthwick, V., Schmidt, S., Piazolo, S., Gundlach, C., Griera, A., Bons, P.D., Jessell, M.W., 2012. The application of in-sity 3D X-ray Diffraction in annealing experiments: FIrst interpretation of substructure development in deformed NaCl. Mater. Sci. Forum 715-716, 461-466. doi:10.4028/www.scientific.net/MSF.715-716.461

Britton, T.B., Wilkinson, A.J., 2012. Stress fields and geometrically necessary dislocation density distributions near the head of a blocked slip band. Acta Mater. 60, 5773-5782. doi:10.1016/j.actamat.2012.07.004

Brown, D.W., Balogh, L., Byler, D., Hefferan, C.M., Hunter, J.F., Kenesei, P., Li, S.F., Lind, J., Niezgoda, S.R., Suter, R.M., 2014. Demonstration of Near Field High Energy X-Ray Diffraction Microscopy on High-Z Ceramic Nuclear Fuel Material. Mater. Sci. Forum 777, 112-117. doi:10.4028/www.scientific.net/MSF.777.112

Brown, D.W., Beyerlein, I.J., Sisneros, T.A., Clausen, B., Tom??, C.N., 2012. Role of twinning and slip during compressive deformation of beryllium as a function of strain rate. Int. J. Plast. 29, 120-135. doi:10.1016/j.ijplas.2011.08.006

Cerrone, A., Stein, C., Pokharel, R., Hefferan, C., Lind, J., Tucker, H., Suter, R., Rollett, A., Ingraffea, A., 2015. Implementation and verification of a microstructure-based capability for modeling microcrack nucleation in LSHR at room temperature. Model. Simul. Mater. Sci. Eng. 23, 35006. doi:10.1088/0965-0393/23/3/035006

Chatterjee, K., Venkataraman, A., Garbaciak, T., Rotella, J., Sangid, M.D., Beaudoin, A., 2015. Study of grain-level deformation and residual stresses in Ti-7Al 
under combined bending and tension using high energy x-ray diffraction ( HEXD ). Int. J. Solids Struct. 94-95, 1-23. doi:10.1016/j.ijsolstr.2016.05.010

Chen, P., Mao, S.C., Liu, Y., Wang, F., Zhang, Y.F., Zhang, Z., Han, X.D., 2013. In-situ EBSD study of the active slip systems and lattice rotation behavior of surface grains in aluminum alloy during tensile deformation. Mater. Sci. Eng. A 580, 114-124. doi:10.1016/j.msea.2013.05.046

Cizek, P., Bai, F., Palmiere, E.J., Rainforth, W.M., 2005. EBSD study of the orientation dependence of substructure characteristics in a model Fe$30 \mathrm{wt} \% \mathrm{Ni}$ alloy subjected to hot deformation. J. Microsc. 217, 138-51. doi:10.1111/j.1365-2818.2005.01429.x

Crumbach, M., Pomana, G., Wagner, P., Gottstein, G., 2001. A Taylor type deformation texture model considering grain interaction and material properties. Part I - Fundamentals, in: Gottstein, G., Molodov, D. (Eds.), Recrystallization and Grain Growth, Vols 1 and 2. Springer-Verlag Berlin, Berlin, pp. 1053-1060.

Dake, J.M., Oddershede, J., Henning, O.S., Werz, T., Shatto, J.C., Uesugi, K., 2016. Direct observation of grain rotations during coarsening of a semisolid $\mathrm{Al}$ $\mathrm{Cu}$ alloy. PNAS PLUS. doi:10.1073/pnas.1602293113

Daniels, J.E., Majkut, M., Cao, Q., Schmidt, S., Wright, J.P., Oddershede, J., 2016. Heterogeneous grain-scale response in ferroic polycrystals under electric field. Sci. Rep. 6, 22820. doi:10.1038/srep22820

Delannay, L., Barnett, M.R., 2012. Modelling the combined effect of grain size and grain shape on plastic anisotropy of metals. Int. J. Plast. 32-33, 70-84. doi:10.1016/j.ijplas.2011.12.002

Di Gioacchino, F., Quinta Da Fonseca, J., 2015. An experimental study of the polycrystalline plasticity of austenitic stainless steel. Int. J. Plast. 74, 92-109. doi:10.1016/j.ijplas.2015.05.012

Eisenlohr, P., Diehl, M., Lebensohn, R.A., Roters, F., 2013. A spectral method solution to crystal elasto-viscoplasticity at finite strains. Int. J. Plast. 46, 3753. doi:10.1016/j.ijplas.2012.09.012

Guery, A., Hild, F., Latourte, F., Roux, S., 2016. Slip activities in polycrystals determined by coupling DIC measurements with crystal plasticity calculations. Int. J. Plast. 81, 249-266. doi:10.1016/j.ijplas.2016.01.008

Hall, S.A., Wright, J., 2015. Three-dimensional experimental granular mechanics. Geotech. Lett. 5, 236-242. doi:10.1680/jgele.15.00094

Hansen, N., Huang, X., Pantleon, W., Winther, G., 2006. Grain orientation and dislocation patterns. Philos. Mag. 86, 3981-3994.

Hedström, P., Han, T.-S., Lienert, U., Almer, J., Odén, M., 2010. Load partitioning between single bulk grains in a two-phase duplex stainless steel during tensile loading. Acta Mater. 58, 734-744.

Hefferan, C.M., Li, S.F., Lind, J., Lienert, U., Rollett, A.D., Wynblatt, P., Suter, R.M., 2009. Statistics of High Purity Nickel Microstructure From High Energy Xray Diffraction Microscopy. Comput. Mater. Contin. 14, 209-219.

Hong, C.S., Huang, X., Winther, G., 2013. Dislocation content of geometrically necessary boundaries aligned with slip planes in rolled aluminium. Philos. Mag. 93, 3118-3141. doi:10.1080/14786435.2013.805270

Huang, X., Winther, G., 2007. Dislocation structures. Part I. Grain orientation dependence. Philos. Mag. 87, 5189-5214.

doi:10.1080/14786430701652851 
Juul, N.Y., Winther, G., Dale, D., Koker, M.K.A., Shade, P., Oddershede, J., 2016. Elastic interaction between twins during tensile deformation of austenitic stainless steel. Scr. Mater. 120, 1-4. doi:10.1016/j.scriptamat.2016.03.022

Kanjarla, A.K., Van Houtte, P., Delannay, L., 2010. Assessment of plastic heterogeneity in grain interaction models using crystal plasticity finite element method. Int. J. Plast. 26, 1220-1233. doi:10.1016/j.ijplas.2009.05.005

Knezevic, M., McCabe, R.J., Tom??, C.N., Lebensohn, R.A., Chen, S.R., Cady, C.M., Gray, G.T., Mihaila, B., 2013. Modeling mechanical response and texture evolution of ??-uranium as a function of strain rate and temperature using polycrystal plasticity. Int. J. Plast. 43, 70-84. doi:10.1016/j.ijplas.2012.10.011

Kocks, U.F., Chandra, H., 1982. Slip geometry in partially contrained deformation. Acta Metall. 30, 695-709.

Krog-Pedersen, S., Bowen, J.R., Pantleon, W., 2009. Quantitative characterization of the orientation spread within individual grains in copper after tensile deformation. Int. J. Mater. Res. 100, 433-438.

Labiche, J.C., Mathon, O., Pascarelli, S., Newton, M. a., Ferre, G.G., Curfs, C., Vaughan, G., Homs, A., Carreiras, D.F., 2007. Invited article: The fast readout low noise camera as a versatile $\mathrm{x}$-ray detector for time resolved dispersive extended x-ray absorption fine structure and diffraction studies of dynamic problems in materials science, chemistry, and catalysis. Rev. Sci. Instrum. 78, 91301. doi:10.1063/1.2783112

Le, G.M., Godfrey, A., Hansen, N., Liu, W., Winther, G., Huang, X., 2013. Influence of grain size in the near-micrometre regime on the deformation microstructure in aluminium. Acta Mater. 61, 7072-7086. doi:10.1016/j.actamat.2013.07.046

Le, G.M., Godfrey, A., Hong, C.S., Huang, X., Winther, G., 2012. Orientation dependence of the deformation microstructure in compressed aluminum. Scr. Mater. 66, 359-362. doi:10.1016/j.scriptamat.2011.11.034

Lebensohn, R.A., Kanjarla, A.K., Eisenlohr, P., 2012. An elasto-viscoplastic formulation based on fast Fourier transforms for the prediction of micromechanical fields in polycrystalline materials. Int. J. Plast. 32-33, 5969. doi:10.1016/j.ijplas.2011.12.005

Lebensohn, R.A., Tomé, C., 1993. A self-consistent anisotropic approach for the simulation of plastic deformation and texture development of polycrystals: Application to zirconium alloys. Acta Metall. Mater. 41, 2611-2624.

Lebensohn, R.A., Zecevic, M., Knezevic, M., McCabe, R.J., 2016. Average intragranular misorientation trends in polycrystalline materials predicted by a viscoplastic self-consistent approach. Acta Mater. 104, 228-236. doi:10.1016/j.actamat.2015.10.035

Lebensohn, R.A., Zecevic, M., Knezevic, M., McCabe, R.J., 2016. Average intragranular misorientation trends in polycrystalline materials predicted by a viscoplastic self-consistent approach. Acta Mater. 104, 228-236. doi:10.1016/j.actamat.2015.10.035

Leffers, T., 1979. A modified Sachs approach to the plastic deformation of polycrystals as a realistic alternative to the Taylor model, in: Haasen, P., Gerold, V., Kostorz, G. (Eds.), 5th International Conference on Strength of Metals and Alloys. Pergamon Press, Aachen, pp. 769-774. 
Li, S., Donohue, B.R., Kalidindi, S.R., 2008. A crystal plasticity finite element analysis of cross-grain deformation heterogeneity in equal channel angular extrusion and its implications for texture evolution. Mater. Sci. Eng. A 480, 17-23. doi:10.1016/j.msea.2007.06.073

Li, S.F., Lind, J., Hefferan, C.M., Pokharel, R., Lienert, U., Rollett, A.D., Suter, R.M., 2013. Three-dimensional plastic response in polycrystalline copper via near-field. J. Appl. Crystallogr. 45, 1098-1108. doi:10.1107/S0021889812039519

Li, S.F., Suter, R.M., 2013. Adaptive reconstruction method for three- dimensional orientation imaging. J. Appl. Crystallogr. 46, 512-524. doi:10.1107/S0021889813005268

Liberman, E.J., Rollett, A.D., Lebensohn, R.A., Kober, E.M., 2015. Calculation of grain boundary normals directly from 3D microstructure images. Model. Simul. Mater. Sci. Eng. 23, 35005. doi:10.1088/0965-0393/23/3/035005

Lin, F.X., Godfrey, A., Jensen, D.J., Winther, G., 2010. 3D EBSD characterization of deformation structures in commercial purity aluminum. Mater. Charact. 61, 1203-1210.

Lind, J., Li, S.F., Pokharel, R., Lienert, U., Rollett, A.D., Suter, R.M., 2014. Tensile twin nucleation events coupled to neighboring slip observed in three dimensions. Acta Mater. 76, 213-220. doi:10.1016/j.actamat.2014.04.050

Ma, A., Roters, F., Raabe, D., 2004. Numerical study of textures and Lankford values for FCC polycrystals by use of a modified Taylor model. Comput. Mater. Sci. 29, 353-361.

Majkut, M., Daniels, J., Wright, J., Schmidt, S., Oddershede, J., 2016. ElectroMechanical Response of Polycrystalline Barium Titanate Resolved at the Grain-Scale. J. Am. Ceram. Soc. accepted. doi:10.1111/jace.14481

Mánik, T., Holmedal, B., 2013. Additional relaxations in the Alamel texture model. Mater. Sci. Eng. A 580, 349-354. doi:10.1016/j.msea.2013.05.071

Margulies, L., Winther, G., Poulsen, H.F., 2001. In situ measurement of grain rotation during deformation of polycrystals. Science 291, 2392-2394. doi:10.1126/science.1057956

Martin, G., Sinclair, C.W., Lebensohn, R.A., 2014. Microscale plastic strain heterogeneity in slip dominated deformation of magnesium alloy containing rare earth. Mater. Sci. Eng. A 603, 37-51. doi:10.1016/j.msea.2014.01.102

McCabe, R.J., Misra, A., Mitchell, T.E., 2004. Experimentally determined content of a geometrically necessary dislocation boundary in copper. Acta Mater. 52, 705-714.

Molinari, A., Canova, G.R., Ahzi, S., 1987. A self consistent approach of the large deformation polycrystal viscoplasticity. Acta Metall. 35, 2983-2994.

Nicaise, N., Berbenni, S., Wagner, F., Berveiller, M., Lemoine, X., 2011. Coupled effects of grain size distributions and crystallographic textures on the plastic behaviour of if steels. Int. J. Plast. 27, 232-249. doi:10.1016/j.ijplas.2010.05.001

Nisr, C., Ribárik, G., Ungár, T., Vaughan, G.B.M., Cordier, P., Merkel, S., 2012. High resolution three-dimensional $\mathrm{X}$-ray diffraction study of dislocations in grains of MgGe03 post-perovskite at $90 \mathrm{GPa}$. J. Geophys. Res. Solid Earth 117, 1-13. doi:10.1029/2011JB008401

Nisr, C., Ribárik, G., Ungár, T., Vaughan, G.B.M., Merkel, S., 2014. Threedimensional X-ray diffraction in the diamond anvil cell: application to 
stishovite. High Press. Res. 7959, 1-9. doi:10.1080/08957959.2014.885021

Obstalecki, M., Wong, S.L., Dawson, P.R., Miller, M.P., 2014. Quantitative analysis of crystal scale deformation heterogeneity during cyclic plasticity using high-energy X-ray diffraction and finite-element simulation. Acta Mater. 75, 259-272. doi:10.1016/j.actamat.2014.04.059

Oddershede, J., Camin, B., Schmidt, S., Mikkelsen, L.P., Sørensen, H.O., Lienert, U., Poulsen, H.F., Reimers, W., 2012. Measuring the stress field around an evolving crack in tensile deformed Mg AZ31 using three-dimensional X-ray diffraction. Acta Mater. 60, 3570-3580. doi:10.1016/j.actamat.2012.02.054

Oddershede, J., Majkut, M., Cao, Q., Schmidt, S., Wright, J.P., Kenesei, P., Daniels, J.E., 2015. Quantitative grain-scale ferroic domain volume fractions and domain switching strains from three-dimensional X-ray diffraction data. J. Appl. Crystallogr. 48, 882-889. doi:10.1107/S1600576715007669

Oddershede, J., Schmidt, S., Poulsen, H.F., Sørensen, H.O., Wright, J., Reimers, W., 2010. Determining grain resolved stresses in polycrystalline materials using three-dimensional X-ray diffraction. J. Appl. Crystallogr. 43, 539-549. doi:10.1107/S0021889810012963

Oddershede, J., Wright, J.P., Beaudoin, A., Winther, G., 2015. Deformation-induced orientation spread in individual bulk grains of an interstitial-free steel. Acta Mater. 85, 301-313. doi:10.1016/j.actamat.2014.11.038

Offerman, S.E.E., van Dijk, N.H., Sietsma, J., Lauridsen, E.M., Margulies, L., Grigull, S., Poulsen, H.F., van der Zwaag, S., Dijk, N. van, 2006. Phase transformations in steel studied by 3DXRD microscopy. Nucl. Instruments ... 246, 194-200. doi:10.1016/j.nimb.2005.12.058

Ozturk, T., Stein, C., Pokharel, R., Hefferan, C., Tucker, H., Jha, S., John, R., Lebensohn, R.A., Kenesei, P., Suter, R.M., Rollett, A.D., 2016. Simulation domain size requirements for elastic response of 3D polycrystalline materials. Model. Simul. Mater. Sci. Eng. 24, 15006. doi:10.1088/09650393/24/1/015006

Pagan, D.C., Miller, M.P., 2014. Connecting heterogeneous single slip to diffraction peak evolution in high-energy monochromatic X-ray experiments. J. Appl. Crystallogr. 47, 887-898. doi:10.1107/S1600576714005779

Pokharel, R., Lind, J., Kanjarla, A.K., Lebensohn, R.A., Li, S.F., Kenesei, P., Suter, R.M., Rollett, A.D., 2014. Polycrystal Plasticity : Comparison Between GrainScale Observations of Deformation and Simulations. Annu. Rev. Condens. Matter Phys. 5, 317-346. doi:10.1146/annurev-conmatphys-031113133846

Pokharel, R., Lind, J., Li, S.F., Kenesei, P., Lebensohn, R.A., Suter, R.M., Rollett, A.D., 2015. In situ Observation of Bulk 3D Grain Evolution During Plastic Deformation in Polycrystalline Cu. Int. J. Plast. 67, 217-234. doi:10.1016/j.ijplas.2014.10.013

Poulsen, H.F., Margulies, L., Schmidt, S., Winther, G., 2003. Lattice rotations of individual bulk grains. Part I: 3D X-ray Characterization. Acta Mater. 51, 3821-3830.

Poulsen, H.F., Nielsen, S.F., Lauridsen, E.M., Schmidt, S., Suter, R.M., 2001. Threedimensional maps of grain boundaries and the stress state of individual grains in polycrystals and powders. J. Appl. Crystallogr. 34, 751-756. doi:10.1107/S0021889801014273

Poulsen, S.O., Lauridsen, E.M., Lyckegaard, a., Oddershede, J., Gundlach, C., Curfs, 
C., Juul Jensen, D., 2011. In situ measurements of growth rates and grainaveraged activation energies of individual grains during recrystallization of 50\% cold-rolled aluminium. Scr. Mater. 64, 1003-1006. doi:10.1016/j.scriptamat.2011.01.046

Quey, R., Driver, J.H., Dawson, P.R., 2015. Intra-grain orientation distributions in hot-deformed aluminium : Orientation dependence and relation to deformation mechanisms. J. Mech. Phys. Solids 84, 506-527.

Quey, R., Piot, D., Driver, J.H., 2010. Microtexture tracking in hot-deformed polycrystalline aluminium: Experimental results. Acta Mater. 58, 16291642.

Raabe, D., Sachtleber, M., Zhao, Z., Roters, F., Zaefferer, S., 2001. Micromechanical and macromechanical effects in grain scale polycrystal plasticity experimentation and simulation. Acta Mater. 49, 3433-3441.

Robert, W., Piot, D., Driver, J.H., 2004. A rapid deformation texture model incorporating grain interactions. Acta Mater. 50, 1215-1219.

Rollett, A.D., Rohrer, G.S., Suter, R.M., 2015. Understanding materials microstructure and behavior at the mesoscale. MRS Bull. 40, 951-960. doi: $10.1557 / \mathrm{mrs} .2015 .262$

Rosa, A.D., Hilairet, N., Ghosh, S., Garbarino, G., Jacobs, J., Perrillat, J.-P., Vaughan, G., Merkel, S., 2015. In situ monitoring of phase transformation microstructures at Earth's mantle pressure and temperature using multigrain XRD. J. Appl. Crystallogr. 48. doi:10.1107/S1600576715012765

Roters, F., Eisenlohr, P., Hantcherli, L., Tjahjanto, D.D., Bieler, T.R., Raabe, D., 2010. Overview of constitutive laws, kinematics, homogenization and multiscale methods in crystal plasticity finite-element modeling: Theory, experiments, applications. Acta Mater. 58, 1152-1211. doi:10.1016/j.actamat.2009.10.058

Sachs, G., 1928. Zur Ableitung einer Fliessbedingung. Z. Ver. Deu. Ing. 72, 734736.

Sarma, G.B., Dawson, P.R., 1996. Effects of interactions among crystals on the inhomogenous deformations of polycrystals. Acta Mater. 44, 1937-1953.

Saxena, A.K., Tewari, A., Pant, P., 2015. Quantitative analysis of orientation and near neighbor interaction effects during deformation of polycrystalline Ti6Al4V. Mater. Sci. Eng. A 648, 1-8. doi:10.1016/j.msea.2015.09.013

Schmidt, S., 2014. GrainSpotter: a fast and robust polycrystalline indexing algorithm. J. Appl. Crystallogr. 47, 276-284. doi:10.1107/S1600576713030185

Schmidt, S., Nielsen, S.F., Gundlach, C., Margulies, L., Huang, X., Juul Jensen, D., 2004. Watching the Growth of Bulk Grains During Recrystallization of Deformed Metals. Science (80-. ). 305, 229-232. doi:10.1126/science.1098627

Schmidt, S., Olsen, U.L., Poulsen, H.F., Sørensen, H.O., Lauridsen, E.M., Margulies, L., Maurice, C., Juul Jensen, D., 2008. Direct observation of 3-D grain growth in $\mathrm{Al}-0.1 \%$ Mn. Scr. Mater. 59, 491-494. doi:10.1016/j.scriptamat.2008.04.049

Schuren, J.C., Shade, P.A., Bernier, J. V, Li, S.F., Blank, B., Lind, J., Kenesei, P., Lienert, U., Suter, R.M., Turner, T.J., Dimiduk, D.M., Almer, J., 2015. New opportunities for quantitative tracking of polycrystal responses in three dimensions. Curr. Opin. SOLID STATE Mater. Sci. 19, 235-244. 
doi:10.1016/j.cossms.2014.11.003

Sedmák, P., Pilch, J., Heller, L., Wright, J., Sedlák, P., Frost, M., Sittner, P., 2016. Grain-resolved analysis of localized deformation in nickel-titanium wire under tensile load. Science (80-. ). 353, 559-562.

Sharma, H., Huizenga, R.M., Bytchkov, A., Sietsma, J., Offerman, S.E., 2012. Observation of changing crystal orientations during grain coarsening. Acta Mater. 60, 229-237. doi:10.1016/j.actamat.2011.09.057

Sørensen, H.O., Hakim, S.S., Pedersen, S., Christiansen, B.C., Balogh, Z.I., Hem, C.P., Pasarin, I.S., Schmidt, S., Olsen, U.L., Oddershede, J., Frandsen, C., Feidenhans'L, R., Stipp, S.L.S., 2012a. Non-destructive identification of micrometer-scale minerals and their position within a bulk sample. Can. Mineral. 50, 501-509. doi:10.3749/canmin.50.2.501

Sørensen, H.O., Schmidt, S., Wright, J.P., Vaughan, G.B.M., Techert, S., Garman, E.F., Oddershede, J., Davaasambu, J., Paithankar, K.S., Gundlach, C., Poulsen, H.F., 2012b. Multigrain crystallography. Zeitschrift fur Krist. 227, 63-78. doi:10.1524/zkri.2012.1438

Tang, W., Halm, K.L., Trinkle, D.R., Koker, M.K.A., Lienert, U., Kenesei, P., Beaudoin, A.J., 2015. A study of stress relaxation in AZ31 using high-energy X-ray diffraction. Acta Mater. 101, 71-79. doi:10.1016/j.actamat.2015.08.072

Taylor, G.I., 1938. Plastic strain in metals. J. Inst. Met. 62, 307-324.

Toda, H., Kamiko, T., Tanabe, Y., Kobayashi, M., Leclere, D.J., Uesugi, K., Takeuchi, A., Hirayama, K., 2016. Diffraction-amalgamated grain boundary tracking for mapping 3D crystallographic orientation and strain fields during plastic deformation. Acta Mater. 107, 310-324. doi:10.1016/j.actamat.2016.01.072

Vachhani, S.J., Doherty, R.D., Kalidindi, S.R., 2016. Studies of grain boundary regions in deformed polycrystalline aluminum using spherical nanoindentation. Int. J. Plast. 81, 87-101. doi:10.1016/j.ijplas.2016.01.001

Van Houtte, P., Delannay, L., Samajdar, I., 1999. Quantitative Prediction of Cold Rolling Textures in Low-Carbon Steel by Means of the Lamel Model. Textures Microstruct. 31, 109-149. doi:10.1155/TSM.31.109

Van Houtte, P., Li, S., Seefeldt, M., Delannay, L., 2005. Deformation texture prediction: from the Taylor model to the advanced Lamel model. Int. J. Plast. 21, 589-624. doi:10.1016/j.ijplas.2004.04.011

Varlioglu, M., Lienert, U., Park, J.-S., Jones, J.L., Üstündag, E., 2010. Thermal and Electric Field-Dependent Evolution of Domain Structures in Polycrystalline BaTiO 3 Using the 3D-XRD Technique. Texture, Stress. Microstruct. 2010, 910793. doi:10.1155/2010/910793

Wang, L., Lind, J., Phukan, H., Kenesei, P., Park, J.-S., Suter, R.M., Beaudoin, A.J., Bieler, T.R., 2014. Mechanical twinning and detwinning in pure Ti during loading and unloading - An in situ high-energy X-ray diffraction microscopy study. Scr. Mater. 92, 35-38. doi:10.1016/j.scriptamat.2014.08.008

Wang, S., Wang, M.P., Chen, C., Xiao, Z., Jia, Y.L., Li, Z., Wang, Z.X., 2014. Orientation dependence of the dislocation microstructure in compressed body-centered cubic molybdenum. Mater. Charact. 91, 10-18. doi:10.1016/j.matchar.2014.02.001

Wei, Y., Godfrey, A., Liu, W., Liu, Q., Huang, X., Hansen, N., Winther, G., 2011. Dislocations, boundaries and slip systems in cube grains of rolled aluminium. Scr. Mater. 65, 355-358. doi:10.1016/j.scriptamat.2011.05.005 
West, S.S., Schmidt, S., S, H.O., Winther, G., Poulsen, H.F., Margulies, L., Gundlach, C., Jensen, D.J., 2009. Direct non-destructive observation of bulk nucleation in $30 \%$ deformed aluminum. Scr. Mater. 61, 875-878. doi:10.1016/j.scriptamat.2009.07.027

Winther, G., 2008. Slip systems extracted from lattice rotations and dislocation structures. Acta Mater. 56, 1919-1932.

Winther, G., 2005. Effect of grain orientation dependent microstructures on flow stress anisotropy modelling. Scr. Mater. 52, 995-1000.

Winther, G., Hong, C.S., Huang, X., 2015. Low-Energy Dislocation Structure (LEDS) character of dislocation boundaries aligned with slip planes in rolled aluminium. Philos. Mag. 95, 1471-1489. doi:10.1080/14786435.2015.1033488

Winther, G., Huang, X., 2007. Dislocation structures. Part II. Slip system dependence. Philos. Mag. 87, 5215-5235. doi:10.1080/14786430701591505

Winther, G., Margulies, L., Schmidt, S., Poulsen, H.F., 2004. Lattice rotations of individual bulk grains Part II: correlation with initial orientation and model comparison. Acta Mater. 52, 2863-2872. doi:10.1016/j.actamat.2004.02.045

Wong, S.L., Obstalecki, M., Miller, M.P., Dawson, P.R., 2015. Stress and deformation heterogeneity in individual grains within polycrystals subjected to fully reversed cyclic loading. J. Mech. Phys. Solids 79, 157-185. doi:10.1016/j.jmps.2015.03.010

Wong, S.L., Park, J.S., Miller, M.P., Dawson, P.R., 2013. A framework for generating synthetic diffraction images from deforming polycrystals using crystalbased finite element formulations. Comput. Mater. Sci. 77, 456-466. doi:10.1016/j.commatsci.2013.03.019

Wu, G.L., Godfrey, A., Juul-Jensen, D., Liu, Q., 2005. Deformation strain inhomogeneity in columnar grain nickel. Scr. Mater. 53, 565-570.

Xie, Q., Van Bael, A., Sidor, J., Moerman, J., Van Houtte, P., 2014. A new clustertype model for the simulation of textures of polycrystalline metals. Acta Mater. 69, 175-186. doi:10.1016/j.actamat.2014.01.038

Zhang, H., Diehl, M., Roters, F., Raabe, D., 2016. A virtual laboratory using high resolution crystal plasticity simulations to determine the initial yield surface for sheet metal forming operations. Int. J. Plast. 80, 111-138. doi:10.1016/j.ijplas.2016.01.002

Zhang, K., Holmedal, B., Hopperstad, O., Dumoulin, S., Gawad, J., Van Bael, A., Van Houtte, P., 2015. Multi-level modelling of mechanical anisotropy of commercial pure aluminium plate: Crystal plasticity models, advanced yield functions and parameter identification. Int. J. Plast. 66, 3-30. doi:10.1016/j.ijplas.2014.02.003

Zhang, X., Park, J., Almer, J., Li, M., 2015. Characterization of neutron-irradiated HT-UPS steel by high-energy X- ray diffraction microscopy. J. Nucl. Mater. 311. doi:10.1016/j.jnucmat.2015.11.063 


\section{Figures}

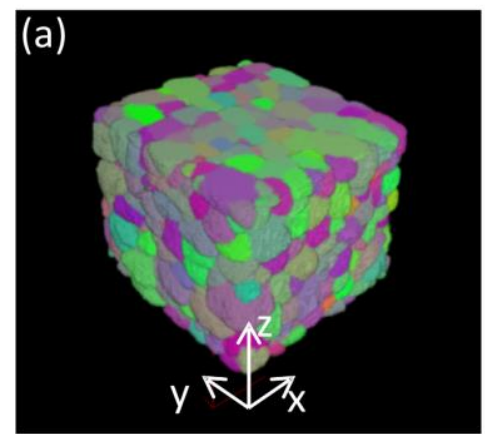

(b)

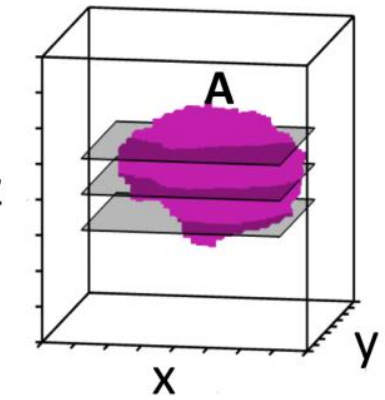

(c)

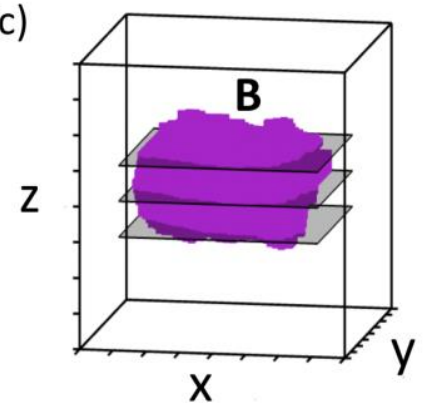

(d)
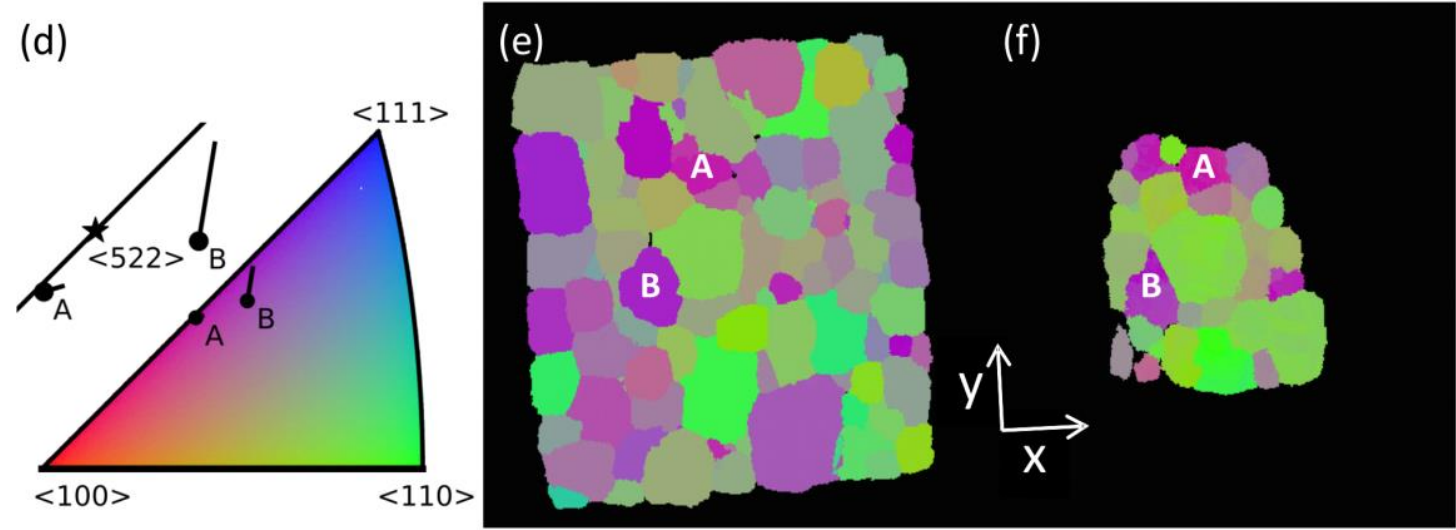

Figure 1 (a) 3D space-filling grain map of IF steel in the undeformed state. The map is $0.7 \times 0.7 \times 0.5 \mathrm{~mm}^{3}$ with a voxel size of $2 \times 2 \times 2 \mu \mathrm{m}^{3}$ and completeness level of $60 \%$. Grains are colour coded according to the crystallographic direction of the tensile $z$ axis as in (d). 3D view of two selected grains, (b) A and (c) B, in boxes with dimensions of $160 \times 160 \times 160 \mu^{3}$. Approximate positions of three slices through the grains are indicated (see Figure 7 and Figure 8). (d) Colour code for grain maps indicating the average orientations of grains $\mathrm{A}$ and $\mathrm{B}$ at $9 \%$ deformation as circles and the rotation paths as lines. 2D slices through the (e) undeformed and (f) deformed maps showing the positions of grains A and B. 

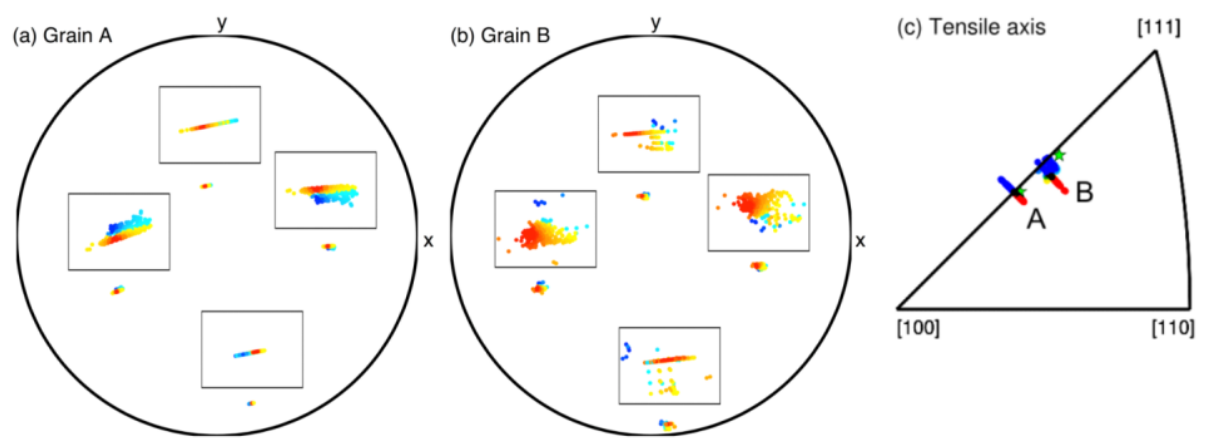

Figure 2 Experimentally determined orientation spread in grains $\mathrm{A}$ and $\mathrm{B}$ at $9 \%$ strain. (a) and (b) $\langle 111\rangle$ pole figures where insets give the orientation spread in higher resolution. (c) Stereographic triangle of the spread of the tensile axis, where green stars mark the initial orientations of the two grains. Red and blue colours in all figures designate different rotation directions of the tensile axis in the stereographic triangle.
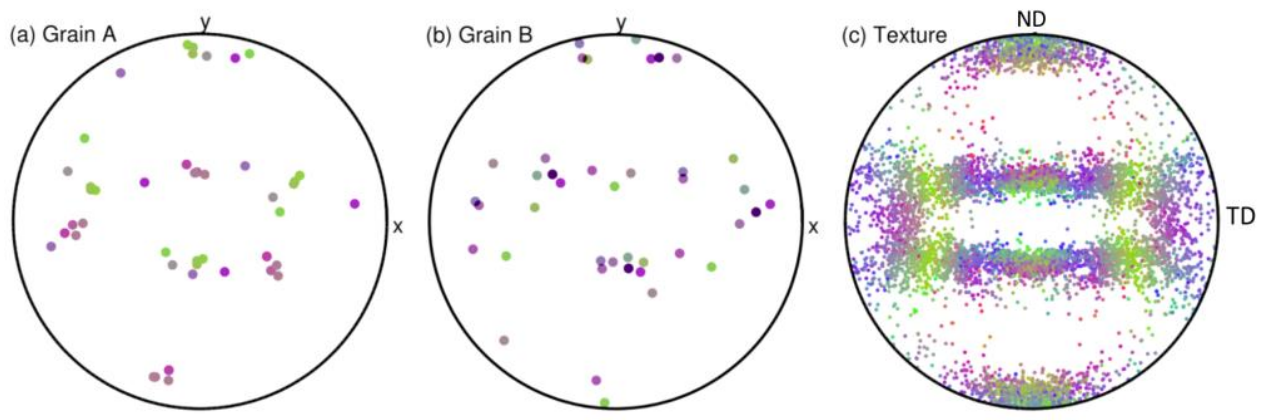

Figure $3<111>$ pole figures showing the undeformed orientations of all immediate neighbours to: (a) grain A, (b) grain B, and (c) all grains in sample.

(a)

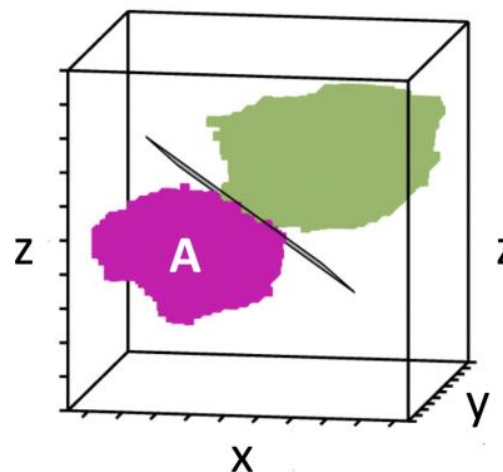

(b)

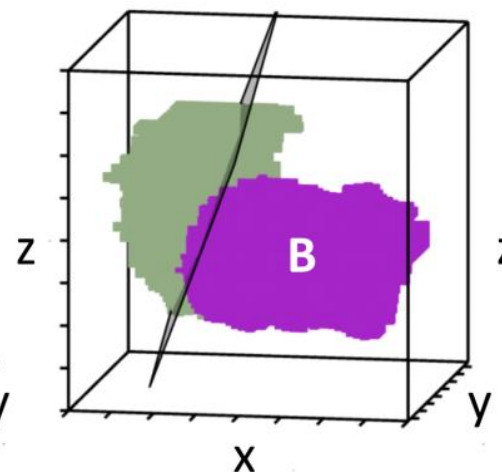

(c)

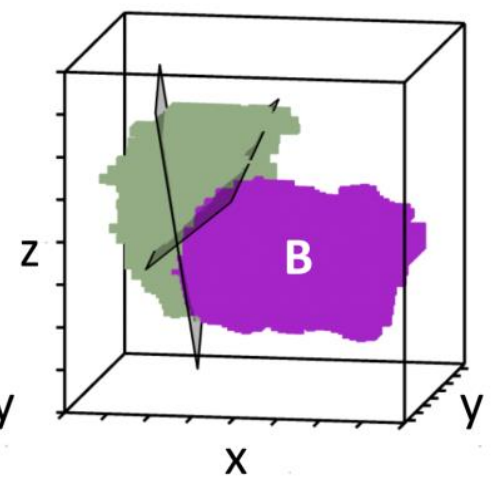

Figure 4 (a) Representative fit of a single boundary plane, illustrated for grain A. (b) A poor fit of a single boundary plane obtained for grain B. (c) Two alternative planes giving a superior description of the grain boundary in (b). Each tick on the axes corresponds to $20 \mu \mathrm{m}$ and colour coding as in Figure $\mathbf{1}$. 
(a)

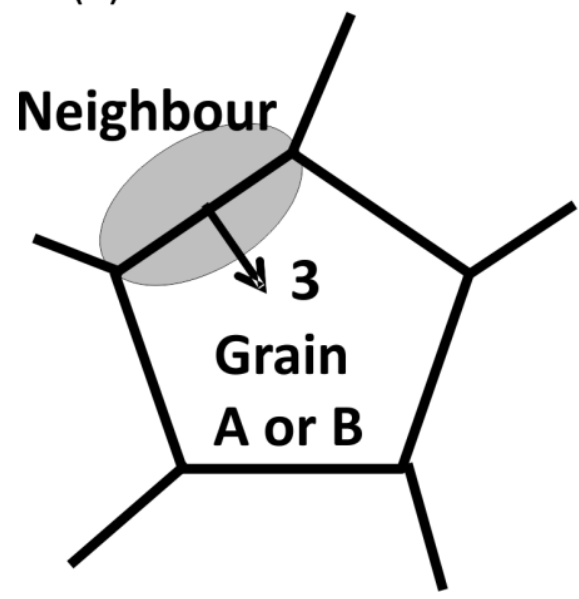

(b)

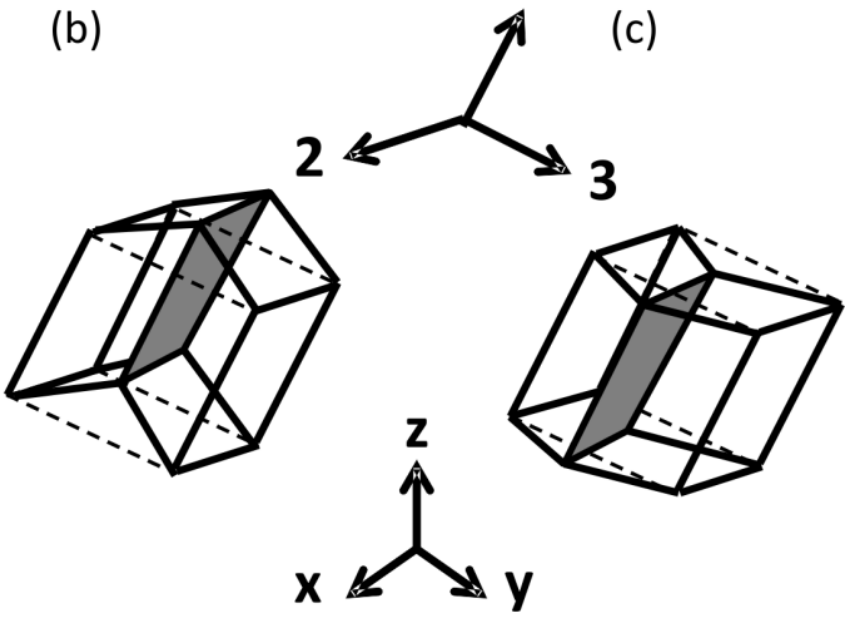

Figure 5 (a) Illustration of interaction in a grain boundary region of unspecified spatial extension as illustrated by the grey ellipse. Schematic of (b) $\varepsilon_{13}$ and (c) $\varepsilon_{23}$ cooperative shears of equal magnitude but opposite signs at the boundary between two grains. The coordinate system $(1,2,3)$ is defined by the grain boundary, with 3 being the grain boundary normal. The coordinate system of the grain boundary may be arbitrarily oriented with respect to the loading axes (x, y, z). (Adapted from (Van Houtte et al., 2005)). 

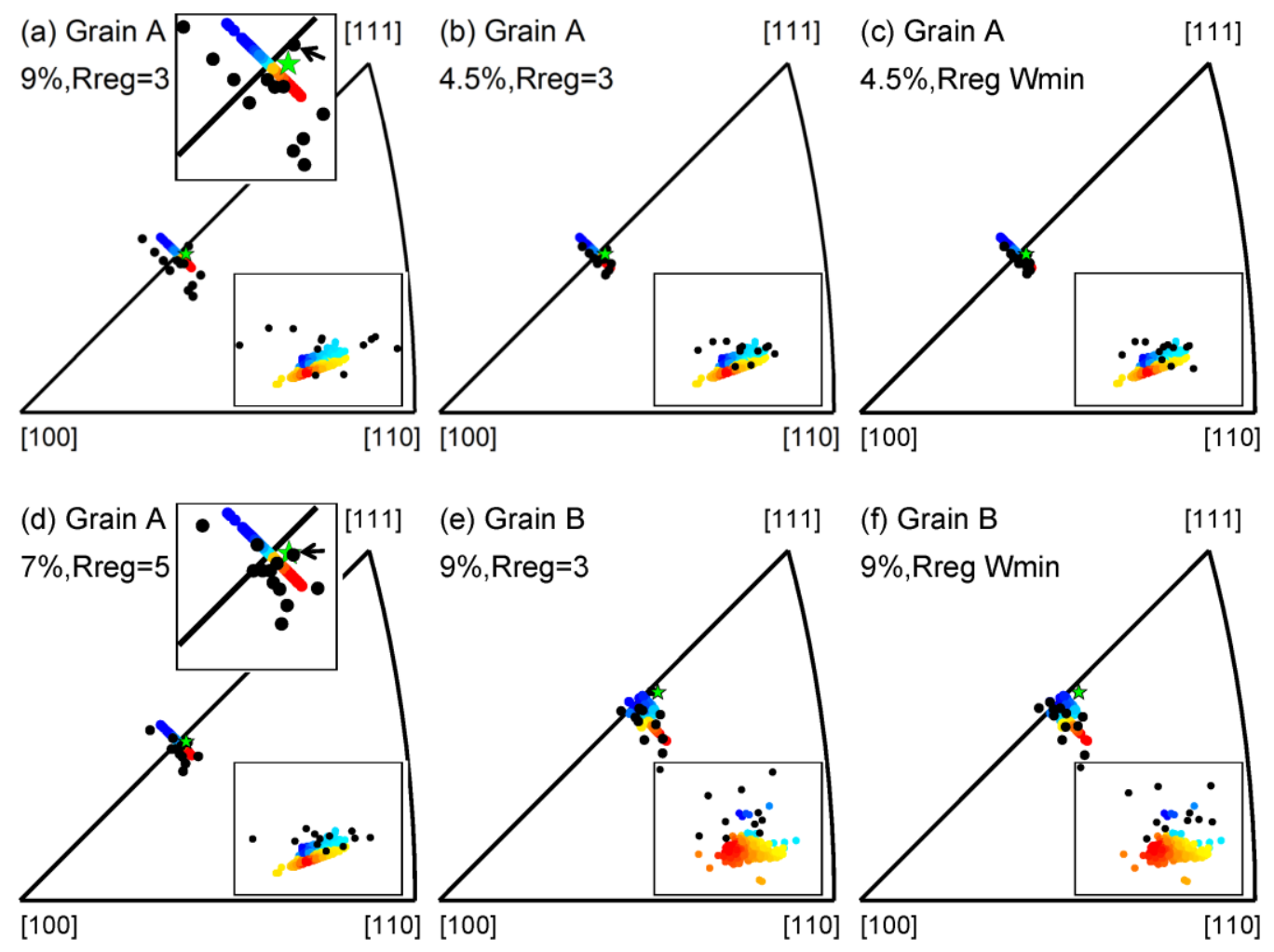

Figure 6 Stereographic triangles representing orientations of the tensile axis for ALAMEL simulations of grain boundary regions (black dots) and measurements (coloured dots, Figure 2), and the spread of one $<111>$ pole as insets inside the triangle. The $<111>$ pole chosen lies far from the tensile axis and thus presents the spread of a direction, which differs significantly from the tensile axis. Green stars mark the initial grain orientation. Predictions are made with different boundary conditions. For grain A: (a) tensile strain of $9 \%, \boldsymbol{R}_{\text {reg }}=3$ (arrow in second inset marks the tensile axis of a region predicted to rotate in a non-observed direction), (b) tensile strain of $4.5 \%, \boldsymbol{R}_{\boldsymbol{r e g}}=3$, (c) tensile strain of $4.5 \%, \boldsymbol{R}_{\boldsymbol{r e g}}$ giving minimum work, (d) tensile strain of $7 \%, \boldsymbol{R}_{\text {reg }}=5$ (arrow in second inset marks the same region as in (a)). For grain B: (e) tensile strain of $9 \%, \boldsymbol{R}_{\boldsymbol{r e g}}=3$, (f) tensile strain of $4.5 \%$, $\boldsymbol{R}_{\text {reg }}$ giving minimum work. 

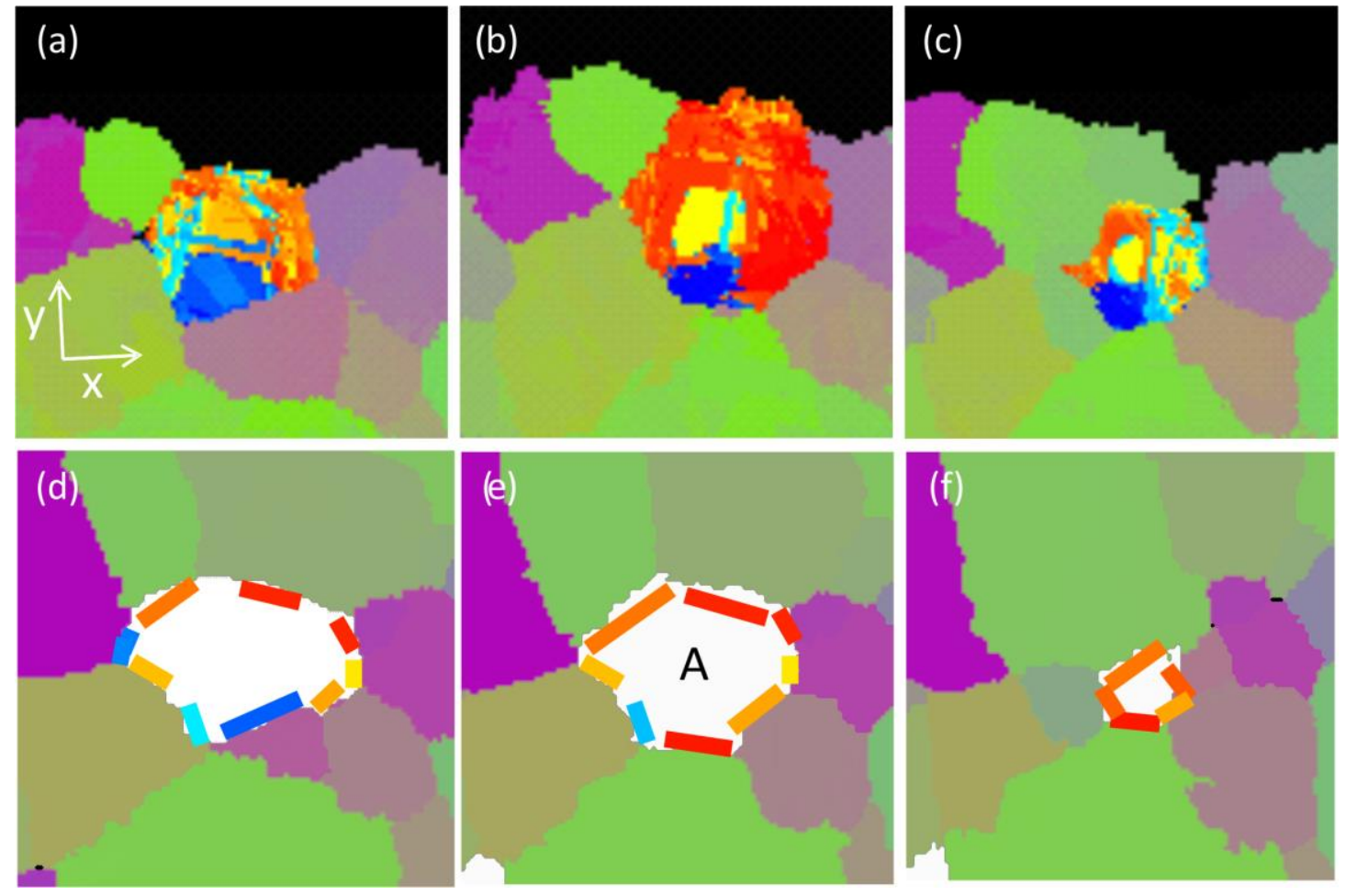

Figure 7 (a)-(c) Three slices through the deformed map of grain A after 9\% deformation. (d)-(f) Predictions of the ALAMEL model after 7\% tensile strain and $\boldsymbol{R}_{\boldsymbol{r e g}}=5$ represented by coloured lines of arbitrary width along the grain boundaries. In order to clearly reveal all neighbours to grain A, the predictions are presented in slices through the undeformed map (see Figure 1(b) for the position of the slices, (d) being the top slice). The colour coding of both experimental and predicted orientations within grain $\mathrm{A}$ is the same as in Figure 2, while the colour coding of the neighbouring grains is according to their tensile axis as in Figure $\mathbf{1}$. 

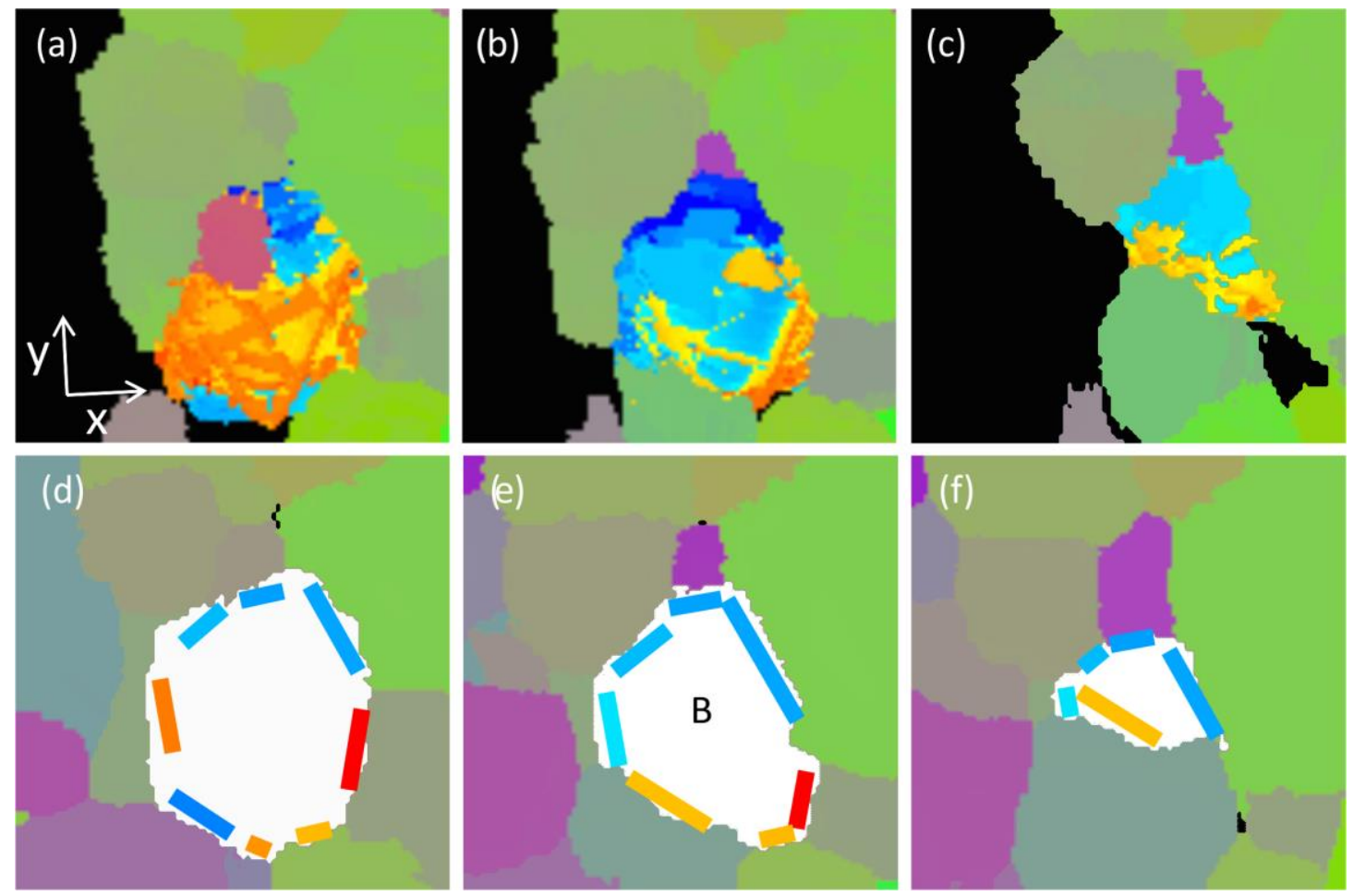

Figure 8 (a)-(c) Three slices through the deformed map of grain B after 9\% deformation. (d)-(f) Predictions of the ALAMEL model after 9\% tensile strain and $\boldsymbol{R}_{\boldsymbol{r e g}}=3$ represented by coloured lines of arbitrary width along the grain boundaries. In order to clearly reveal all neighbours to grain A, the predictions are presented in slices through the undeformed map (see Figure 1(b) for the position of the slices, (d) being the top slice). The colour coding of both experimental and predicted orientations within grain $\mathrm{A}$ is the same as in Figure 2, while the colour coding of the neighbouring grains is according to their tensile axis as in Figure $\mathbf{1}$. 

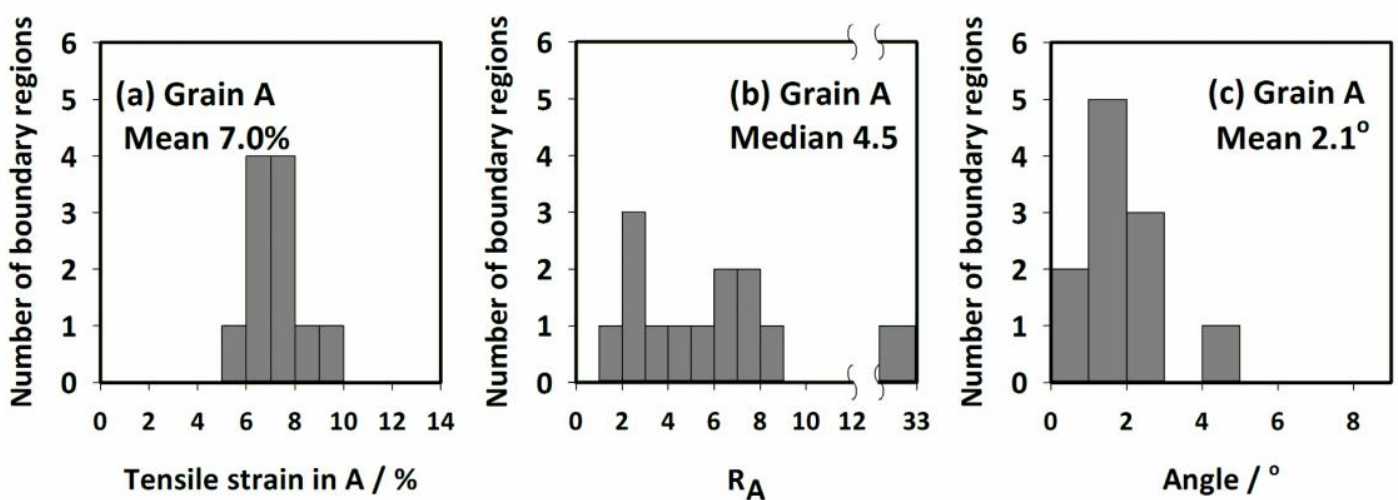

Tensile strain in A / \%

$\mathbf{R}_{\mathbf{A}}$
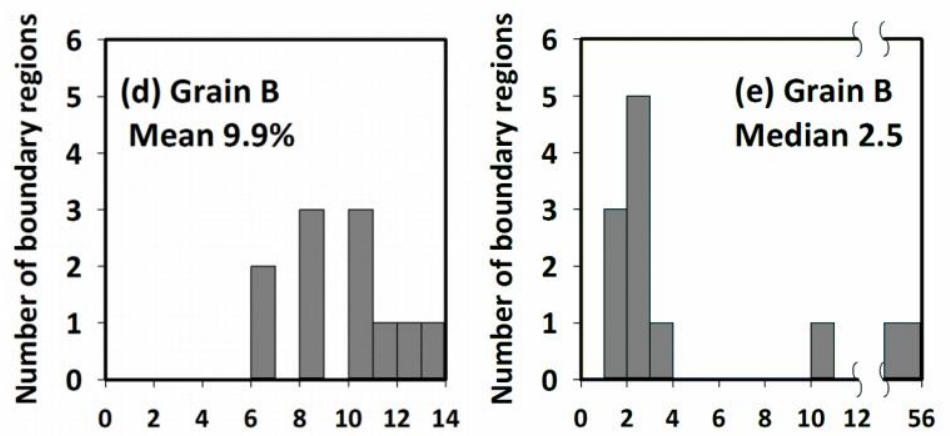

Tensile strain in B / \%

$\mathbf{R}_{\mathbf{B}}$

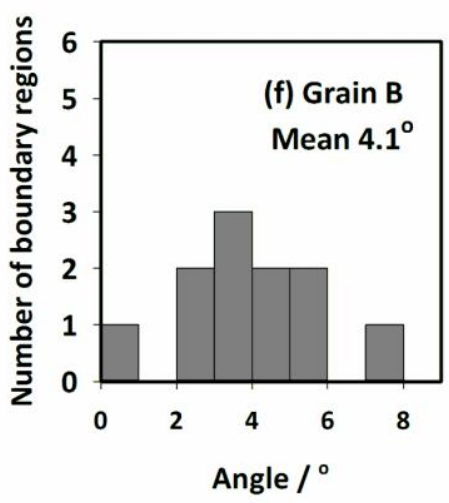

Figure 9 Distribution of selected parameters characterising the predictions from the ALAMEL model at the boundaries of grain A using 7\% elongation, $\boldsymbol{R}_{\text {reg }}=5$ ((a)-(c)), and grain B using $9 \%$ elongation, $\boldsymbol{R}_{\text {reg }}=3$ ((d)-(f)). (a) and (d) tensile strain, (b) and (e) Lankford parameters, and (c) and (f) rotation angles for grain A and B.
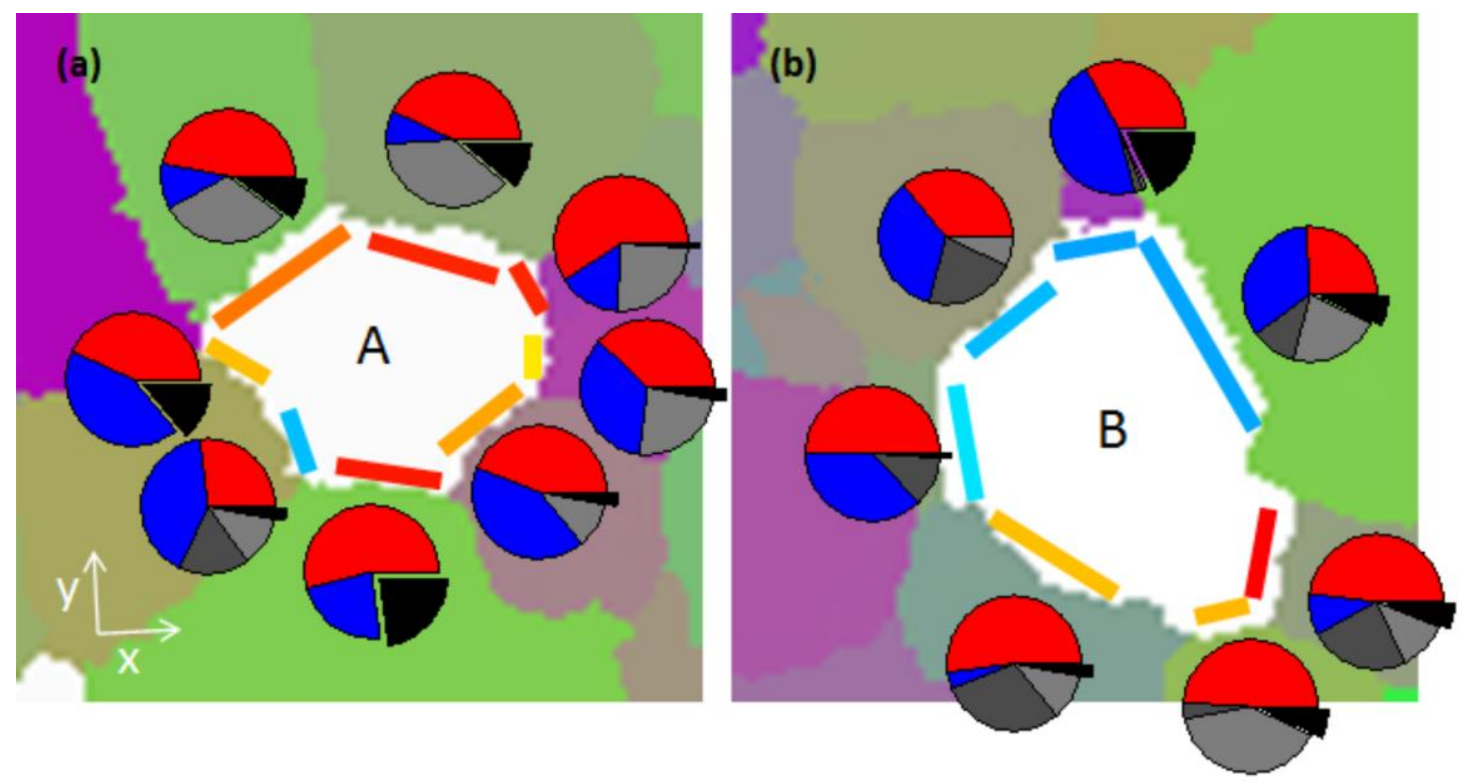

P: $(0 \overline{1} \overline{1})[11 \overline{1}]$ C: $(\overline{1} 01)[111]$ CDP: $(1 \overline{1} 0)[11 \overline{1}]$ CDC: $(1 \overline{1} 0)[\overline{111}] \mathbf{\square}:$ Others

Figure 10 Slip distributions for each grain boundary region in a slice of (a) grain A and (b) grain B. 

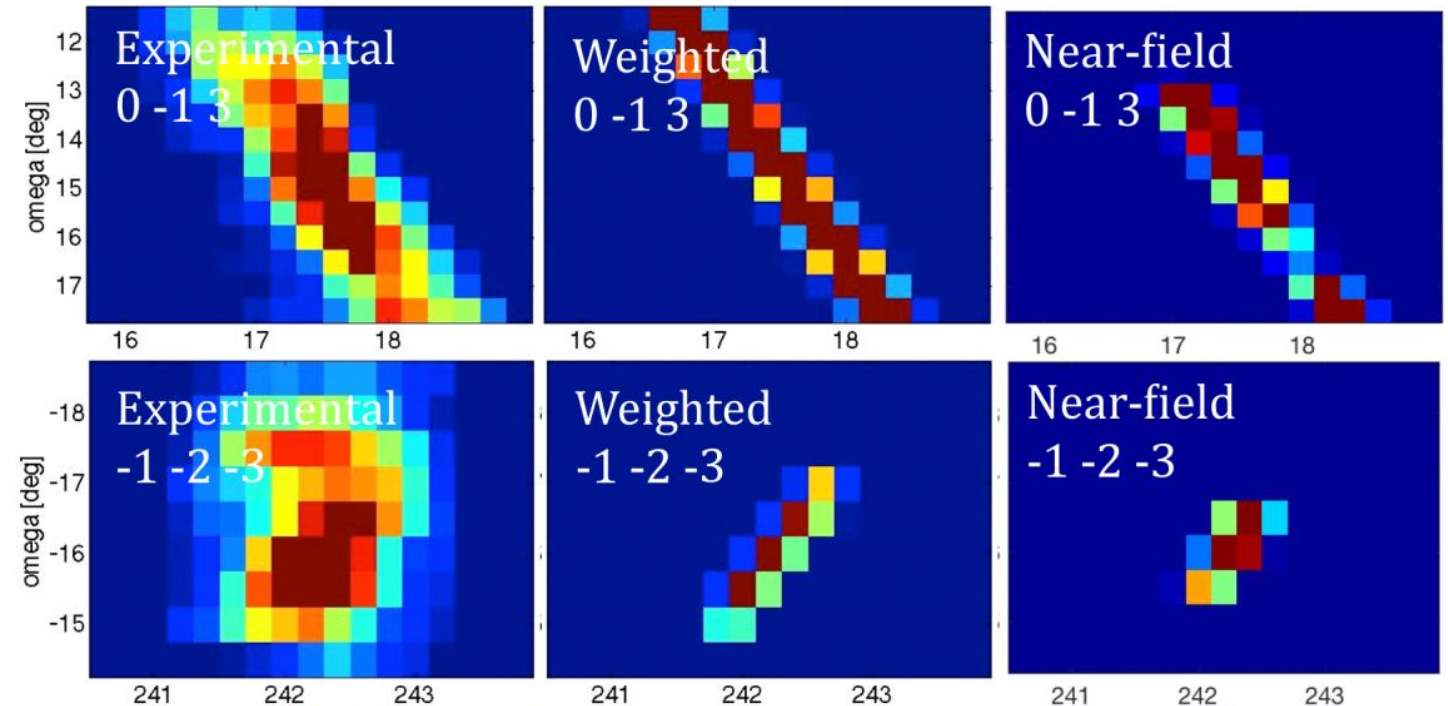
18
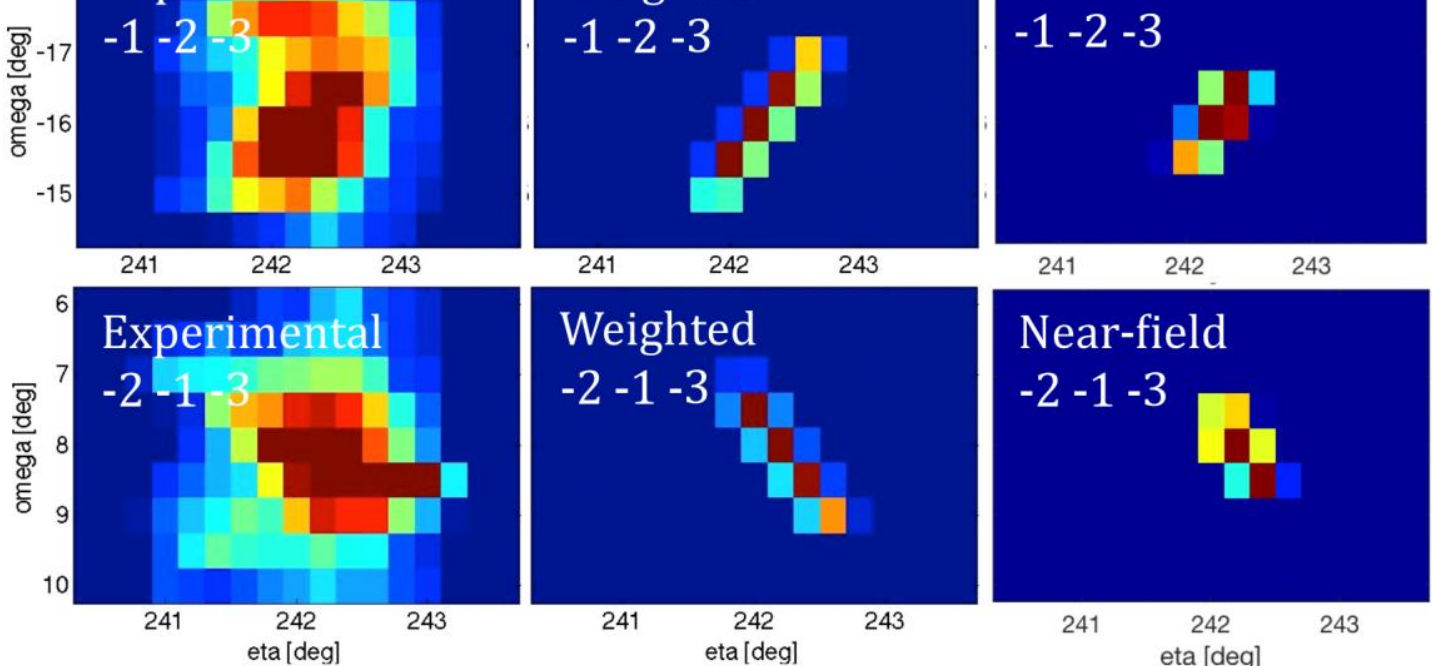

Figure 11 Selected reflections for $10 \mu \mathrm{m}$ slice through grain A after $9 \%$ deformation measured on the far-field detector (left), projected from orientations previously found to approximate the spots (Oddershede et al., 2015) (centre), and projected from the spatially resolved intra-granular orientation distribution determined in the near-field mapping (right). 